\title{
Spectroscopic metallicities of Vega-like stars ${ }^{\star}$
}

\author{
C. Saffe ${ }^{1, \star \star}$, M. Gómez ${ }^{2}$, O. Pintado ${ }^{3}$, and E. González ${ }^{4}$ \\ 1 Complejo Astronómico El Leoncito, CC 467, 5400 San Juan, Argentina \\ e-mail: csaffe@casleo.gov.ar \\ 2 Observatorio Astronómico de Córdoba, Laprida 854, 5000 Córdoba, Argentina \\ e-mail: mercedes@oac.uncor.edu \\ 3 Instituto Superior de Correlación Geológica (INSUGEO), 4000 Tucumán, Argentina \\ e-mail: opintado@tucbbs.com.ar \\ ${ }^{4}$ Facultad de Ciencias Exactas, Físicas y Naturales (UNSJ), 5400 San Juan, Argentina \\ e-mail: erip.p.a.gonzalez@gmail.com
}

Received 26 May 2008 / Accepted 1 August 2008

\section{ABSTRACT}

\begin{abstract}
Aims. We aim to determine the metallicities of 113 Southern Hemisphere Vega-like candidate stars in relation to the giant exoplanet host group and field stars.

Methods. We applied two spectroscopic methods of abundance determinations: equivalent width measurements together with the ATLAS9 model atmospheres and the WIDTH9 program, and a comparison of observed spectra with a grid of synthetic spectra.

Results. For the Vega-like group, the metallicities are indistinguishable from those of field stars not known to be associated with planets or disks. This result is quite different from the metallicities of giant exoplanet host stars, which are metal-rich in comparison to field stars.
\end{abstract}

Key words. techniques: spectroscopic - stars: abundances - stars: late-type

\section{Introduction}

It is well established that stars with giant planets are, on average, metal-rich in comparison to stars that do not harbor Dopplerdetected planets (see, for example, Santos et al. 2004). However, stars that have Neptune-mass planets do not seem to follow the same trend. In other words, Neptune-like or super-Earth planets do not form preferentially around metal-rich stars (e.g. Udry et al. 2006; Sousa et al. 2008). Two hypotheses have been put forward to explain the peculiar high metallicity of the giant exoplanet host stars: a) a primordial origin and b) a pollution of the convective zone of the star. In the first case the "excess" of metallicity was already present in the parent cloud from which the star-bearing planet was formed (see, for example, Santos et al. 2001). In the pollution scenario the convective zone of the star is contaminated by the infall or accretion of planets and/or planetesimals (see, for example, González et al. 2001).

Santos et al. (2004) found a lack of correlation between the thickness of the convective zone and the metallicity for a sample of FG dwarfs with planets. As the convective zone acts as a diluting medium, for a given amount of accreted material, $\mathrm{F}$ dwarfs with thinner convective zones should exhibit a greater degree of pollution than $\mathrm{G}$ dwarfs with thicker zones. On average, $\mathrm{F}$ and $\mathrm{G}$ dwarfs exhibit similar metallicities and the pollution hypothesis is not favored by these observations. The primordial origin of the "excess" remains an alternative to explain the relatively high metallicity of stars with giant planets with respect to field stars.

Pasquini et al. (2007) compared the metallicities of giant and dwarf stars with giant planets and found that the first group

* Tables 1-4 are only available in electronic form at http: //www . aanda.org

$\star \star$ On a fellowship from CONICET, Argentina. has, on average, lower metallicities than the dwarfs. The smaller mass of the convective zone of the dwarfs with respect to the giants provides a plausible explanation for this difference. The diluting effect of the convective zone is efficient for the giants and tends to lower the metallicity to its primordial value. In this case, the pollution scenario is favored (over the primordial origin) since it can explain the observed difference in metallicities between dwarfs and giants with planets. However, Hekker \& Meléndez (2007) do not confirm Pasquini et al. result. In fact the first authors suggest that the metallicity enhancement of stars hosting giant planets might also be valid for giant stars. We note that the samples of stars in both cases are relatively small (14 objects in the case of Pasquini et al. and 20 for Hekker \& Meléndez). The metallicity trend of giant stars with giant planets remains an open issue for the time being.

Even though the origin or the cause of the "excess" of metallicity of stars with giant planets is not well understood, these stars are metal-rich and this is a feature that distinguishes this group from stars with similar physical properties and no giant planets detected.

Vega-like stars are a group of objects that show infrared excesses in their spectral energy distributions that can be attributed to the presence of dust in circumstellar disks. The first members or candidate members of the class were selected by IRAS and had mainly A-F spectral types (Aumann et al. 1984; Gillett 1986; Backman \& Paresce 1993; Sylvester et al. 1996; Mannings \& Barlow 1998; Fajardo-Acosta et al. 1999; Sylvester \& Mannings 2000; Habing et al. 2001; Laureijs et al. 2002; Sheret et al. 2004). Vega ( $\alpha$ Lyr) is one of the four proto-types of the group or the "fabulous four" (Vega, $\beta$ Pictoris, Fomalhaut = $\alpha$ PsA and $\epsilon$ Eridanis; Gillett 1986) and has given the name to the class. 
More recently, Spitzer has contributed to the detection of $\mathrm{G}$ dwarfs with infrared excesses (Meyer et al. 2004; Rieke et al. 2005; Kim et al. 2005; Chen et al. 2005; Uzpen et al. 2005; Beichman et al. 2005, 2006a; Bryden et al. 2006; Silverstone et al. 2006; Su et al. 2006; Trilling et al. 2008). Since the excesses come from distances similar to that of the Kuiper-Belt to the Sun, these stars have also received the designation of KuiperBelt analogs or Kuiper-Belt-like stars. In this paper we adopt the term "Vega-like stars" to refer to both IRAS and Spitzer detections.

The metallicity of Vega-like stars has previously been investigated by Greaves et al. (2006) and Chavero et al. (2006), deriving nearly solar values. However these works analyzed relatively small samples of objects. Greaves et al. (2006) studied a group of 18 FGK Vega-like stars whereas Chavero et al. (2006) included 42 FG dwarfs with infrared excesses in their metallicity determination. In addition these previous works do not include stars of A spectral type which represent the majority of IRAS detections. Greaves et al. (2006) derived their sample from the Doppler searches for planets that in general include solar type stars. Chavero et al. (2006) used Strömgren photometry to determine the metallicity. These works were also restricted to late spectral types.

Both stars with planets and the Vega-like stars have evidence of the presence of circumstellar material, in the form of planet/s, in the first case, or dust in a circumstellar disk, in the second. As mentioned before, the giant exoplanet hosts are metal-rich. This fact may have facilitated the formation of planets (Pollack et al. 1996). In this paper we determine spectroscopic metallicities of a large sample of Vega-like stars and compare our result with the exoplanet host group. We include objects of B-K spectral types, observable from the Southern Hemisphere.

\section{The sample}

We compiled a total of 113 Southern Hemisphere Vega-like candidate stars from the literature, based on their infrared or submillimetric excess emission (Backman \& Paresce 1993; Sylvester et al. 1996; Mannings \& Barlow 1998; Fajardo-Acosta et al. 1999; Sylvester \& Mannings 2000; Habing et al. 2001; Laureijs et al. 2002; Sheret et al. 2004). This compilation also includes $\mathrm{G}$ dwarfs with infrared excess recently detected by Spitzer (Beichman et al. 2005, 2006a; Bryden et al. 2006; Su et al. 2006; Trilling et al. 2008). Specifically the list comprises objects with BAFGK spectral types (22, 38, 28, 17 and 8, respectively). All the stars are luminosity class V (Hipparcos catalogue) and have distances between 5 and 300 pc. Table 1 (available in the online version of the paper) lists the observed objects, including the $v \sin i$ values from the literature (Glebocki et al. 2000; Mora et al. 2001; Yudin 2001; Royer et al. 2002; Cutispoto et al. 2002, 2003; Pizzolato et al. 2003; Strom et al. 2005; Reiners 2006).

Table 1 includes a sub-sample of stars that were originally selected by IRAS as candidate Vega-like stars. However, when observed by Spitzer the infrared excesses were deemed to be of little significance. These objects are: HD 10800, HD 20794, HD 38393, HD 41700, HD 68456, HD 160691, HD 169830, HD 203608, and HD 216437 (Beichman et al. 2005, 2006a; Bryden et al. 2006; Hillenbrand et al. 2008; Trilling et al. 2008). For example, Bryden et al. (2006) found that for HD 10800 $\frac{f_{\text {MIPS_70 mum }}}{f_{*}}=1.3$ (the observed flux over the photospheric emission at $70 \mu \mathrm{m})$ and $\frac{f_{\text {MIPS_70 mum }}}{f_{*}}=1.2$ for HD 68456. This group of objects should be considered with caution.

\section{Observations and data reduction}

The stellar spectra were obtained at the Complejo Astronomico El Leoncito (CASLEO), using the Jorge Sahade 2.15-m telescope equipped with a REOSC echelle spectrograph ${ }^{1}$ and a TEK $1024 \times 1024$ CCD detector. The REOSC spectrograph uses gratings as cross dispensers. We used a grating with 400 lines $\mathrm{mm}^{-1}$, covering the spectral range $\lambda \lambda 3500-6500$, giving a resolving power of $\sim 12500$. Three individual spectra for each object were obtained in four observing runs: August 0508, 2005, August 18-22, 2005, February 18-25, 2006 and May $04-07,2007$ and have $S / N$ ratio of about 300.

The spectra were reduced using IRAF $^{2}$ standard procedures for echelle spectra. We applied bias and flat corrections and then normalized order by order with the continuum task, using 7-9 order Chebyshev polynomials. We also corrected the scattered light in the spectrograph (apscatter task). We fitted the background with a linear function on both sides of the echelle apertures, using the task apall. The resolution of the reduced spectra is $0.17 \AA / \mathrm{pix}$.

\section{Metallicity determinations}

We used two different methods of abundance determination:

1) Fe line equivalent width measurements together with the ATLAS9 (Kurucz 1993) model atmosphere corresponding to a given star and the WIDTH9 ${ }^{3}$ program;

2) a comparison of the observed and synthetic spectra using the Downhill method (Gray et al. 2001). In particular we used the grid of synthetic spectra calculated by Munari et al. (2005). This method offers the advantage that there is no need to identify and measure the equivalent widths of many Fe lines as with the WIDTH9 program.

\subsection{Metallicity determinations using the WIDTH program}

To determine abundances by this method it is necessary to estimate the stellar parameters $T_{\text {eff }}$ and $\log g$, by means of the Strömgren photometry, for example. With these quantities we adopt the Kurucz (1993) model atmosphere appropriate for each star. The model that initially is chosen has solar metallicity. Finally the Kurucz model together with the measured equivalent widths are used by the WIDTH9 program (Kurucz 1992, 1993) to derive the metallicity.

To obtain $T_{\text {eff }}$ and $\log g$, we have used the $u v b y \beta$ mean colors of Hauck \& Mermilliod (1998) with two different calibrations: Napiwotzki et al. (1993) and Castelli et al. (1997) and Castelli (1998) (hereafter N93 and C97, respectively), with the TEMPLOGG code (Rogers et al. 1995). This program has been used in the COROT mission preparation (see, for example, Lastennet et al. 2001; Guillon \& Magain 2006) and includes reddening corrections, according to Domingo \& Figueras (1999), for stars in the range A3-F0, and to Nissen (1988) for spectral types F0-G2.

We have compared the temperatures and gravities derived using both calibrations (N93 and C97) and noticed some differences, particularly in the later parameter. For this reason we

\footnotetext{
${ }^{1}$ On loan from the Institute d'Astrophysique de Liege, Belgium.

2 IRAF is distributed by the National Optical Astronomical Observatories which is operated by the Association of Universities for Research in Astronomy, Inc., under a cooperative agreement with the National Science Foundation.

${ }^{3}$ http://kurucz . harvard. edu/programs.html
} 
initially determined metallicities using values derived from both calibrations and later on considered if they significantly affect the final metallicity values. We have also compared the obtained $T_{\text {eff }}$ with those published by Nordström et al. (2004). We found a good agreement, in particular with the N93 calibration. With the values of $T_{\text {eff }}$ and $\log g$ derived for each object, we have chosen the corresponding model atmosphere using the Kurucz ATLAS9 (Kurucz 1993) code.

The stellar lines were identified using multiplet tables (Moore 1945), wavelengths and transition probabilities (Reader et al. 1980), as well as more specialized references for the Fe II lines (Johansson 1978). The Fe lines used are listed in the Table 2 (available in the online version of the paper), along with the corresponding $\log g f$ values from Fuhr et al. (1988) and Kurucz $(1988,1994)$. This list includes lines usually measured for earlytype stars (e.g. Qiu et al. 2001; Saffe \& Levato 2004) as well as solar-type stars (e.g. González 1998; González et al. 2001). The equivalent widths were measured by fitting Gaussian profiles through the stellar metallic lines using the IRAS splot task. There is no more than a $15 \%$ difference among the equivalent widths of the same line, measured in different spectra. We have excluded from our abundance determinations very blended lines.

To determine the abundances we need an initial estimation of the microturbulent velocity $(\xi)$. For this estimation we have used the standard method. We computed the abundances from the Fe lines for a range of possible values of $\xi$ satisfying two conditions: a) that the abundances of $\mathrm{Fe}$ lines is not dependent on the equivalent widths and $b$ ) that the rms errors are minima. To achieve the first condition the slope in the plot abundance vs. $\xi$ must be zero. We tried different $\xi$ values to fulfill this requirement. The final abundance value should fulfill both conditions a) and b). In this sense the abundance and microturbulent velocity determinations are recursive and simultaneous. Once a $\xi$ value has been fixed the abundances corresponding to all chemical species measured are determined using the WIDTH9 code.

The WIDTH9 code requires the model atmosphere calculated by the ATLAS9 program, the equivalent width of each line as well as atomic constants such as oscillator strength $(\log g f)$ values, excitation potentials, damping constants, etc. This code calculates the theoretical equivalent widths for an initial input abundance and compares these values with the measured equivalent widths. Then the code modifies the abundance to achieve a difference between theoretical and measured equivalent widths $<0.01 \mathrm{m \AA}$. The final values of the metallicities corresponding to the N93 and C97 calibrations, are listed in Table 3 (available in the online version of the paper). In this table we also include the fundamental stellar parameters $T_{\text {eff }}, \log g$ and $\xi$, corresponding to each calibration, the slope of the plot $[\mathrm{Fe} / \mathrm{H}]$ vs. equivalent width, the number of lines used in each determination, as well as the rms of the average.

To estimate errors for our WIDTH metallicities we consider the following facts. The most significant contribution to the final uncertainties probably comes from the equivalent width measurements. We assume a $5 \%$ error due to the continuum level determination. This translates into $20 \%$ maximum uncertainties in the metallicity estimation. The atomic constants may also have uncertainties. In particular we estimate that the oscillator strength values may cause differences of about $10 \%$ in the calculated metallicity. Finally to provide an estimation of "typical" errors introduced by the WIDTH method we increased the $T_{\text {eff }}$ by $150 \mathrm{~K}$ and the $\log g f$ by 0.15 , and recalculated the metallicity value for each star. We derived a median difference of 0.20 dex. The largest difference corresponds to HD 28978 (0.55 dex).

\subsection{Metallicity derivations from synthetic spectra: the Downhill method}

The WIDTH method is not practical when the number of stars is large. For each object, we need to identify and measure many spectral lines. An alternative would be to compare the observed spectra with a grid of synthetic ones corresponding to different values of the metallicites and choose from the grid the spectrum that best reproduces the observed data (Gray et al. 2001). This comparison has the advantage that the complete profiles of the lines and not only the equivalent widths are used in the metallicity determinations.

In general synthetic spectra depend on four parameters: $T_{\text {eff }}$, surface gravity $(\log g)$, metallicity $([\mathrm{Fe} / \mathrm{H}])$ and microturbulent velocity $(\xi)$. Following Gray et al. (2001), we applied a multidimensional Downhill Simplex technique, in which the observed spectrum is compared to a grid of synthetic spectra. The "final" synthetic spectrum is an interpolation of spectra, rather than a single point on the grid. As we are working with four variables $\left(T_{\text {eff }}, \log g,[\mathrm{Fe} / \mathrm{H}]\right.$ and $\xi$ ) the interpolation is done in $4 \mathrm{D}$, minimizing the square differences in each wavelength (i.e., the $\chi^{2}$ statistics). The stellar parameters are determined with a higher accuracy than the steps in the grid since they correspond to interpolated values.

The grid of synthetic spectra was taken from Munari et al. (2005). The parameter range covered by the grid is the following:

$3500 \mathrm{~K}<T_{\text {eff }}<40000 \mathrm{~K}$, with steps of $250 \mathrm{~K}$,

0.0 dex $<\log g<5.0$ dex, with steps of $0.5 \mathrm{dex}$,

$-2.5 \mathrm{dex}<[\mathrm{Fe} / \mathrm{H}]<0.5 \mathrm{dex}$, with steps of $0.5 \mathrm{dex}$,

and $\xi$ values of $0,1,2$, and $4 \mathrm{~km} \mathrm{~s}^{-1}$.

In addition to these parameters, the synthetic spectra are calculated for 15 different rotation velocities, ranging from $0-500 \mathrm{~km} \mathrm{~s}^{-1}$. In all, Munari et al. (2005)'s library contains 625000 different spectra. These authors calculated the complete synthetic spectral library for four resolving powers: 20000, 11500 (GAIA), 8500 (RAVE) and 2000 (SLOAN). Dr. U. Munari kindly provided a grid corresponding to the REOSC/CASLEO resolving power (12500).

Synthetic spectral lines were convolved with the instrumental line profile corresponding to the REOSC/CASLEO. Finally they were also convolved with a Gaussian profile corresponding to the rotational velocities of the sample stars, taken from the literature (Glebocki et al. 2000; Mora et al. 2001; Yudin 2001; Royer et al. 2002; Cutispoto et al. 2002, 2003; Pizzolato et al. 2003; Strom et al. 2005; Reiners 2006). We weighted the synthetic spectra by the blaze function of each of the REOSC spectrograph orders. Finally we normalized and re-sampled our data to compare them with Munari et al. (2005)'s grid. The spectral sampling of the synthetic spectra is $0.02 \AA$.

We have implemented the Downhill method (Gray et al. 2001) by means of a Fortran program. From the stellar spectral type or the Strömgren photometry it is possible to estimate "a starting point" in the 4D grid. The Downhill method provides a searching algorithm within the 4D grid and finds the best match, minimizing the $\chi^{2}$. In our case, the final spectrum is obtained by an interpolation of 16 spectra from Munari et al. (2005)'s grid. In general it takes 15-20 min for each star (50-60 iterations) with a Pentium IV $2.0 \mathrm{GHz}$ processor to find the best interpolated spectrum. Table 4 (available in the online version of the paper) lists the metallicities obtained with the Downhill method for our sample of Vega-like stars. 

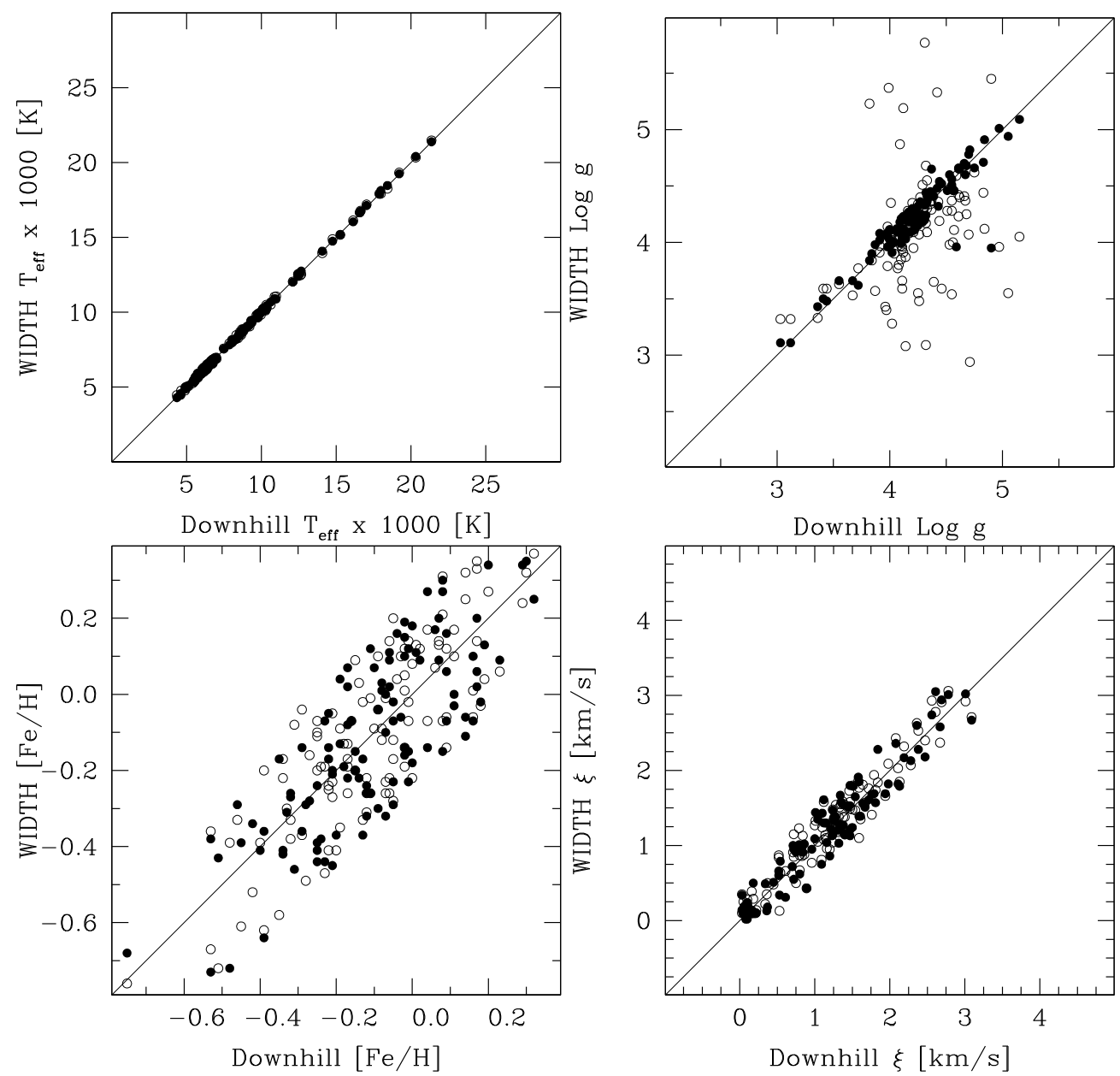

Fig. 1. Comparison of $T_{\text {eff }}, \log g,[\mathrm{Fe} / \mathrm{H}]$, and $\xi$ derived applying the Downhill and the WIDTH methods with the N93 and C97 calibrations. The $x$-axis show values derived from the Downhill and the $y$-axis the WIDTH determined parameters. With filled circles we indicate values obtained using the N93 calibration. The empty circles correspond to the C97 photometric relation. In the case of the $T_{\text {eff }}$ plot (upper-left panel), values obtained from both calibrations are almost superimposed.

To estimate the uncertainties in the metallicities obtained by the Downhill method, we carried out a few tests. We first applied this method to 30 synthetic spectra of known metallicities. The median difference between the derived and known metallicities is 0.02 dex.

The internal consistency of the method has been checked by fixing one of the four variables and comparing the resultant metallicities. Fixed values for each variable were obtained, for example, from an adopted calibration:

a) $T_{\text {eff }}$ was taken from the N93 calibration;

b) $\log g$ was adopted from the N93 calibration;

c) $\xi$ was fixed at $2.9 \mathrm{~km} \mathrm{~s}^{-1}$, the solar value.

The median difference, calculated by fixing 3 of the 4 variables with respect to the "standard" procedure (i.e., with 4 variables), was 0.05 dex. Considering this value and the median difference derived from the comparison with 30 synthetic spectra of known metallicities $(0.02$ dex $)$, we estimate a "typical" uncertainty of $\left(0.02^{2}+0.05^{2}\right)^{-1 / 2} \sim 0.06$ dex for the metallicities derived by the Downhill method.

We finally mention two parameters taken as fixed by the Downhill method, the radial and the rotational velocities. Radial velocities are initially determined minimizing the $\chi^{2}$ with an accuracy of $0.1 \mathrm{~km} \mathrm{~s}^{-1}$ or a median value of 0.03 dex in metallicity. Rotational velocities $(v \sin i)$ from the literature have "typical" dispersions of 5-10\%, corresponding to an error of about $10 \%$ in metallicity.

In summary, we have estimated an internal uncertainty of 0.06 dex for metallicities derived from the Downhill method. A more conservative estimation would indicate a value of $0.1 \mathrm{dex}$. This corresponds to half of the uncertainty calculated for the WIDTH method (0.2 dex). In this manner, the Downhill method allows a more precise determination of the metallicities for our sample of Vega-like objects.

\subsection{Comparison of metallicity determinations by the WIDTH and the Downhill methods}

As mentioned in the previous section we used two calibrations (N93 and C97) with the WIDTH and the Downhill methods. In Fig. 1 we compare the corresponding values of $T_{\text {eff }}, \log g$, $[\mathrm{Fe} / \mathrm{H}]$, and $\xi$. The $x$-axis shows the Downhill derived values and the $y$-axis the WIDTH determinations. In this case filled circles indicate values obtained applying the N93 calibration, while open circles the C97 relation. In general we find a good agreement with the exception of the $\log g$ values. In addition the $\log g$ Downhill method derived values agree better with those obtained with the WIDTH method and the N93 calibration rather than 
Table 5. Medians and dispersions of the metallicities for the Vega-like sample.

\begin{tabular}{lccc}
\hline \hline Method & $\begin{array}{c}\text { Median } \\
{[\mathrm{Fe} / \mathrm{H}]}\end{array}$ & $\begin{array}{c}\text { Dispersion } \\
{[\mathrm{Fe} / \mathrm{H}]}\end{array}$ & $N$ \\
\hline WIDTH+N93 & -0.14 & 0.28 & 113 \\
WIDTH+C97 & -0.11 & 0.26 & 113 \\
Downhill & -0.11 & 0.27 & 113 \\
\hline
\end{tabular}

Note - N93: Napiwotzki et al. (1993)'s calibration; C97: Castelli et al. (1997) and Castelli (1998)'s calibration.

with those determined with the WIDTH and the N97 photometric relation.

Table 5 lists the medians and the dispersions of the metallicities derived by applying the WIDTH and the Downhill methods for the Vega-like group. In the case of the WIDTH method we present the results corresponding to the two calibrations used (N93 and C97). The derived median values are practically indistinguishable.

Figure 2 compares the metallicity distributions calculated with the WIDTH method plus the N93 calibration (histogram shaded at 0 degrees) and the C97 calibrations (histogram shaded at 45 degrees), respectively. The empty histogram shows the distribution derived with the Downhill method for the Vega-like sample. Vertical lines indicate the medians of each distribution. The left line corresponds to the WIDTH+N93 median, and the right line shows (superimposed) the WIDTH+C97 and Downhill medians (see Table 5). The KS-test (Press 1992) indicates that these distributions are similar and represent the same parent population.

We finally adopt the metallicities calculated with the Downhill method for the sample of Vega-like stars, as these determinations use the complete line profiles and not only the equivalent widths. In addition "typical" uncertainties are smaller than those estimated for the WIDTH method.

Figure 3 shows the $T_{\text {eff }}, \log g,[\mathrm{Fe} / \mathrm{H}]$ and $\xi$ values derived in the present contribution ( $x$ axis) and those obtained from the literature ( $y$ axis). In particular we compare our determinations with the four recent works. The open squares correspond to Santos et al. $(2004,2005)$, filled circles to Nordström et al. (2004), empty circles to Fischer \& Valenti (2005), and crosses to Sousa et al. (2008). We note a general good agreement although we also find a moderate dispersion between our determinations and those from the literature. We find no obvious systematic tendencies, except for $0.09 \mathrm{dex}$ in $[\mathrm{Fe} / \mathrm{H}]$ between our estimations and the Fischer \& Valenti (2005) data.

We also noticed a systematic difference of $\sim 0.09$ dex between the Nordström et al. (2004) and Fischer \& Valenti (2005) determinations. The Fischer \& Valenti (2005) determinations are, on average, larger than those from Nordström et al. (2004). Our Downhill method derived metallicities show a better agreement with the Nordström et al. (2004) values than with Fischer \& Valenti (2005). However this later comparison is based on a relatively small number of common stars. Our sample has $24 \mathrm{ob}-$ jects in common with Fischer \& Valenti (2005) and 51 with Nordström et al. (2004). In the work of Nordström et al. (2004) the metallicities are derived as a secondary parameter obtained photometrically. In the case of Fischer \& Valenti (2005), the metallicities are obtained by a comparison with synthetic spectra but using only a small range of wavelengths (6000-6200 ̊).

The works of Santos et al. (2004, 2005) and Sousa et al. (2008) are based on high-signal to-noise spectra covering a wide spectral range. We find a reasonably good agreement with these

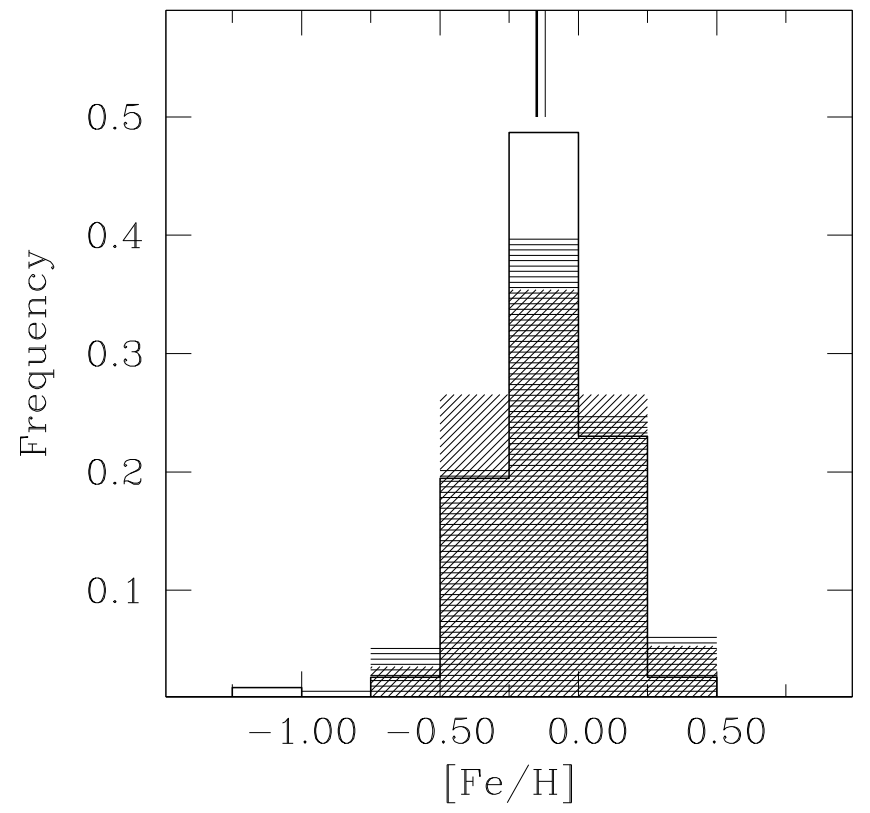

Fig. 2. Metallicity distributions for the Vega-like sample. Histograms shaded at 0 and 45 degrees correspond to the WIDTH method derivations using N93 (Napiwotzki et al. 1993) and C97 (Castelli et al. 1997; Castelli 1998) calibrations, respectively. The empty histogram shows the metallicity distribution derived by the Downhill method. The vertical lines indicate the medians of each distribution. The left line corresponds to the WIDTH+N93 median, and the right line shows (superimposed) the WIDTH+C97 and Downhill medians (see Table 5).

determinations. However this comparison is based on a limited number of common stars: 17 and 12 objects, respectively. Our metallicity values show a slightly better agreement with the Santos et al. data than with the Sousa et al. determinations. In summary, with these limitations and those mentioned in the cases of Nordström et al. (2004) and Fischer \& Valenti (2005) in mind, we consider that the external consistency of the Downhill method derived metallicities is acceptable.

Lines with large equivalent widths are sensitive to NLTE effects. An attempt to analyze such lines in the LTE approach could lead to abundance overestimations, simply due to overestimation of the equivalent widths (e.g. Takeda 1992; Thevenin \& Idiart 1999). In general, equivalent width overestimations become more severe at low temperatures. In addition an apparent systematic difference of abundances between high and low excitation FeI lines $\left([\mathrm{Fe} / \mathrm{H}]_{\text {high }}>[\mathrm{Fe} / \mathrm{H}]_{\text {low }}\right)$ has been attributed to NLTE effects (e.g. Takeda 1992; Ruland et al. 1980). We find no systematic dependence of the WIDTH LTE derived metallicities (and dispersions) on the excitation potential for the stars in our sample, or the atmospheric stellar parameters. Consequently the metallicity scale seems to be constant for all the stars in our sample. However this does not completely rule out NLTE effects. Our LTE calculations, nevertheless, allow us to compare our derivations with most previous works in the literature, which make similar LTE assumptions.

\section{Discussion of the results}

The metallicity of the Solar Neighborhood is usually represented by a control sample of stars, which should exclude, in our case, known Vega-like stars. The selection of the control sample is important, because different groups of objects (i.e., stars selected by different criteria) may have different metallicities. For 

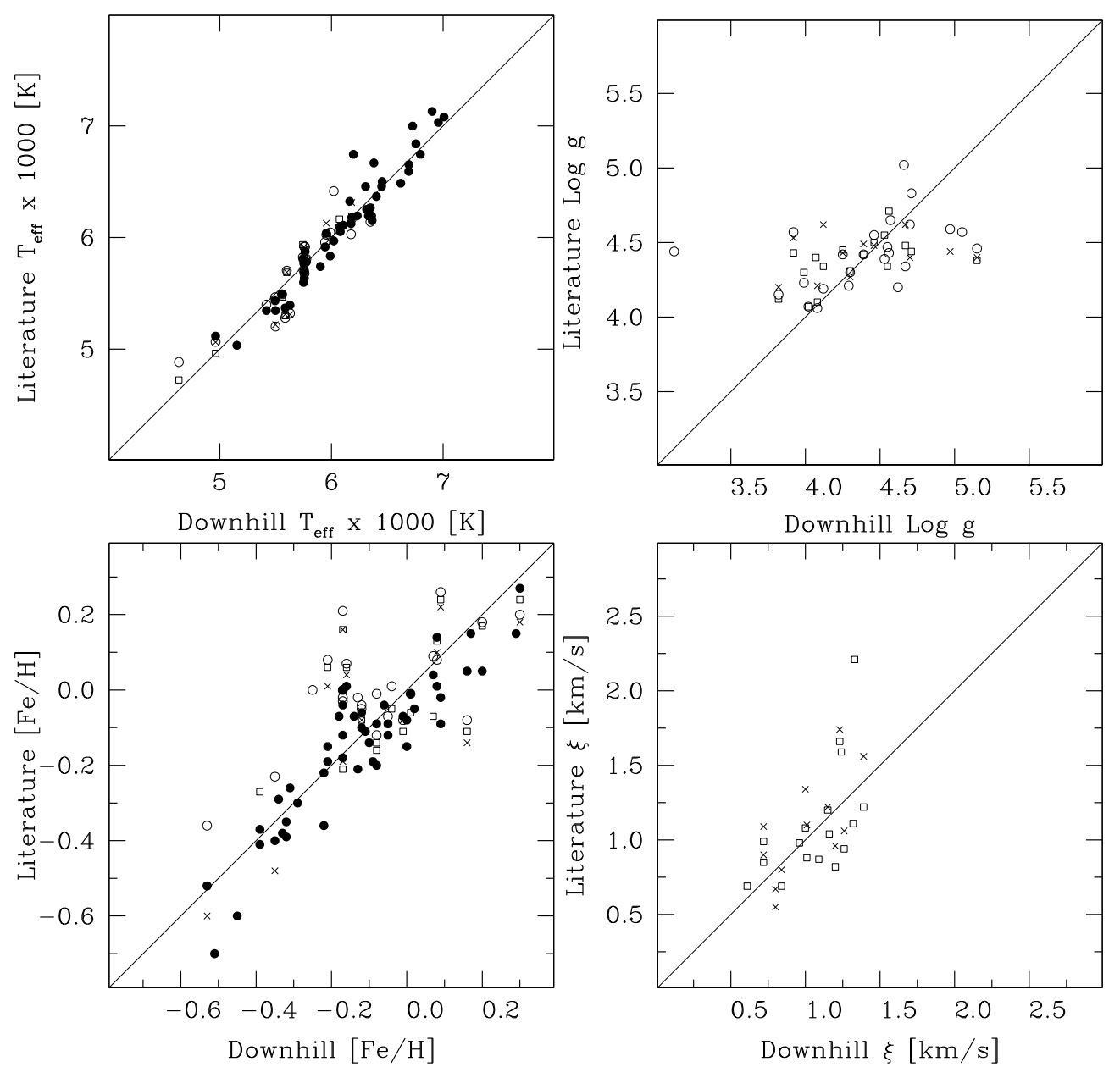

Fig. 3. Comparison of $T_{\text {eff }}, \log g,[\mathrm{Fe} / \mathrm{H}]$ and $\xi$ derived in this contribution ( $x$ axis) with those obtained from the literature ( $y$ axis). The open squares correspond to Santos et al. (2004, 2005), filled circles to Nordström et al. (2004), empty circles to Fischer \& Valenti (2005), and crosses to Sousa et al. (2008).

example, Fischer \& Valenti (2005) compared two different control samples, with the metallicity distribution of exoplanet host stars. Their control sets are volume-limited and magnitudelimited. The medians of the metallicity "excess" of the giant exoplanet host stars compared with the two groups are 0.13 and 0.226 dex, respectively. In other words, the "excess" is real, but the amount depends on the control sample used. The two control sets contain different classes of stars. The magnitudelimited sample includes more massive and metal-rich stars than the volume-limited set.

The metallicity distribution of exoplanet host stars is usually compared with a volume-limited group of solar neighborhood stars (González 1998, 1999; González et al. 2001; Santos et al. 2000, 2003, 2004; Sadakane et al. 2002; Laws et al. 2003). We compared the metallicity distribution of our Vega-like sample with a volume-limited sample of 71 stars, without Doppler detected exoplanets (Santos et al. 2001; Gilli et al. 2006) and with 98 exoplanets host stars (Santos et al. 2004). Metallicity values for these two comparison samples were obtained from Nordström et al. (2004). As discussed in Sect. 4.2, the agreement between our metallicities and those obtained by these authors is acceptable. Figure 4 shows these distributions. Vega-like stars are represented by the empty histogram, stars with giant planets by the histogram shaded at 0 degrees and stars known not to harbor planets detected by the Doppler technique, by the histogram shaded at 45 degrees. The KS test shows no significant difference between the metallicity distributions of the Vega-like stars and stars without planets. On the other hand, the Vega-like stars metallicity distribution is different from the metallicity distribution for stars with giant planets, with a high level of confidence.

Fischer \& Valenti (2005) obtained that the probability that a FGK star harbors a giant planet/s increases as $P(Z) \propto\left(10^{Z}\right)^{2}$, where $Z$ is the stellar metallicity (see also, Wyatt et al. 2007b). If this relation is also applicable to A stars (the bulge of IRAS detected Vega-like stars), the low median value of the metallicity for the Vega-like group $(-0.11 \mathrm{dex}$, see Table 6) indicates that the probably of these stars hosting a planet/s of the type detected by radial velocity surveys is also low. We note, however, that the dispersion of metallicities within the Vega-like stars is also significant $(0.26 \mathrm{dex})$ and at least a fraction of these stars has metallicities high enough to host giant planets, assuming the "excess" of metallicity/presence of a giant planet/s holds for A spectral type stars. In addition Doppler searches do not achieve the required precision to detect giant planets in A stars as metal lines practically disappear.

We also compared the metallicity distribution of Vega-like stars with a sample of 115 stars without excess at 24 or $70 \mu \mathrm{m}$, observed by Spitzer (Beichman et al. 2005, 2006a; Bryden et al. 2006; Su et al. 2006). The metallicities of these stars were taken from literature (Sousa et al. 2008; Santos et al. 2004, 2005; Nordström et al. 2004). Figure 5 shows these distributions. Vega-like stars are indicated by the empty histogram whereas the stars without excess at 24 or $70 \mu \mathrm{m}$ are shown by the histogram shaded at 45 degrees. The KS test shows no significant difference 


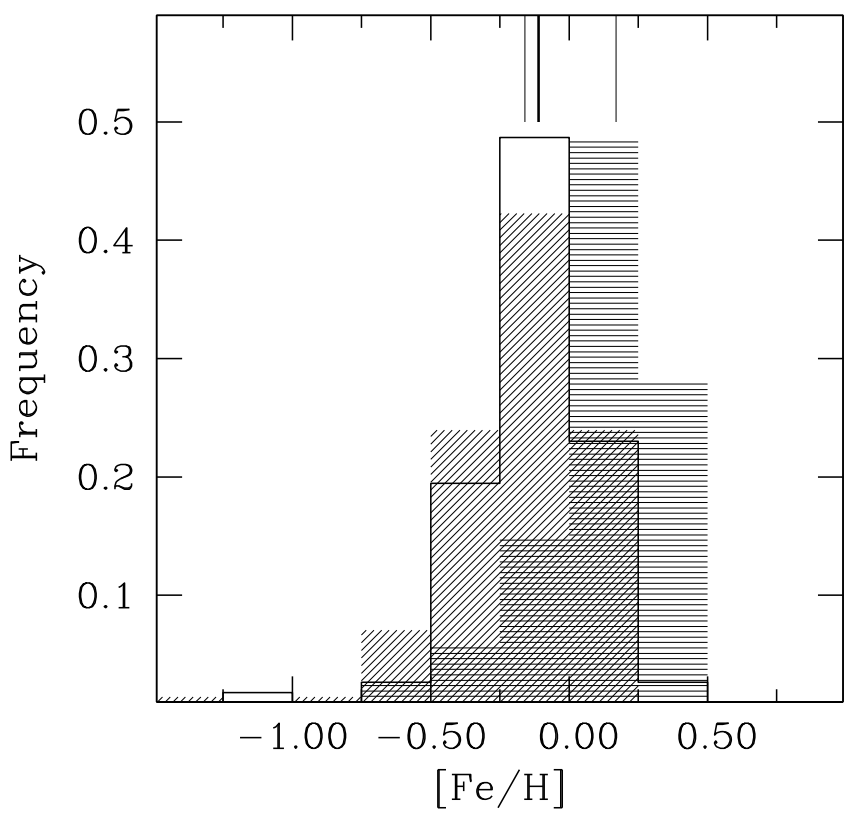

Fig. 4. Metallicity distributions for the Vega-like sample, empty histogram, for stars with giant planets, histogram shaded at 0 degrees, and for stars known not to harbor planets, histogram shaded at 45 degrees. The vertical lines indicate the medians of each distribution: stars without planets, Vega-like stars, and exoplanet host stars, respectively (see Table 6).

Table 6. Medians and dispersions of the Vega-like sample and three comparison groups.

\begin{tabular}{lccc}
\hline \hline Sample & $\begin{array}{c}\text { Median } \\
{[\mathrm{Fe} / \mathrm{H}]}\end{array}$ & $\begin{array}{c}\text { Dispersion } \\
{[\mathrm{Fe} / \mathrm{H}]}\end{array}$ & $N$ \\
\hline Vega-like stars & -0.11 & 0.27 & 113 \\
$\begin{array}{l}\text { Exoplanet host stars } \\
\text { Volume-limited sample }\end{array}$ & +0.17 & 0.22 & 98 \\
$\begin{array}{l}\text { without planets } \\
\text { Stars without excess }\end{array}$ & -0.16 & 0.25 & 71 \\
at 24 or $70 \mu \mathrm{m}$ & -0.12 & 0.24 & 115 \\
\hline
\end{tabular}

between the two distributions. Table 6 lists the medians and the dispersions of the four samples compared in Figs. 4 and 5.

The results in Table 6 indicate that, on average, the Vega-like group has metallicities similar to the stars in the Solar Neighborhood without detected planets or disks, in contrast to the giant exoplanet host stars group. This result confirms and extends previous works by Greaves et al. (2006) and Chavero et al. (2006), based on relatively small numbers of FG Vega-like stars.

In Fig. 6 we analyze the metallicity distribution of Vega-like stars of different spectral types. The number of objects corresponding to each spectral type is indicated between brackets. The vertical bars are the dispersions within the spectral types. A-spectral-type stars still dominate the Vega-like group although Spitzer has significantly contributed $\mathrm{F}$ and $\mathrm{G}$ stars during the last few years (Beichman et al. 2005, 2006a; Bryden et al. 2006; Su et al. 2006). Figure 6 shows no trend of metallicity with spectral type for the Vega-like group.

As suggested by Greaves et al. (2006) the relatively high metallicity of giant exoplanet host stars as well as the solar metallicity value for the Vega-like stars can be understood within the core accumulation model of Pollack et al. (1996). The high

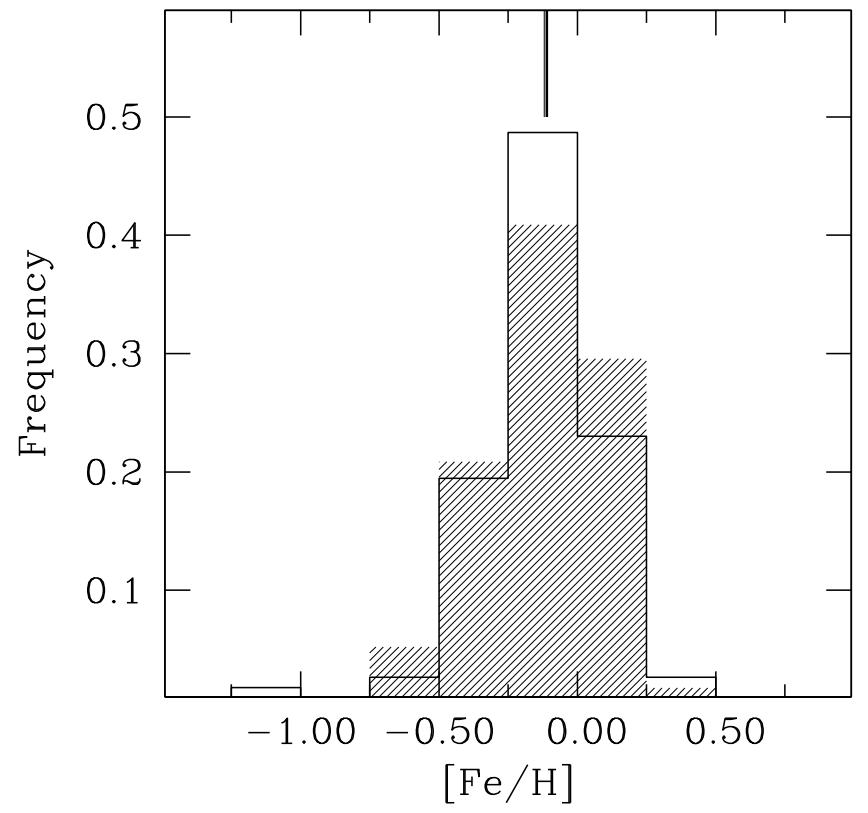

Fig. 5. Metallicity distributions for the Vega-like sample, empty histogram, and for stars without excess at 24 or $70 \mu \mathrm{m}$ (Beichman et al. 2005, 2006a; Bryden et al. 2006; Su et al. 2006). The vertical lines (almost superimposed) indicate the medians of each distribution.

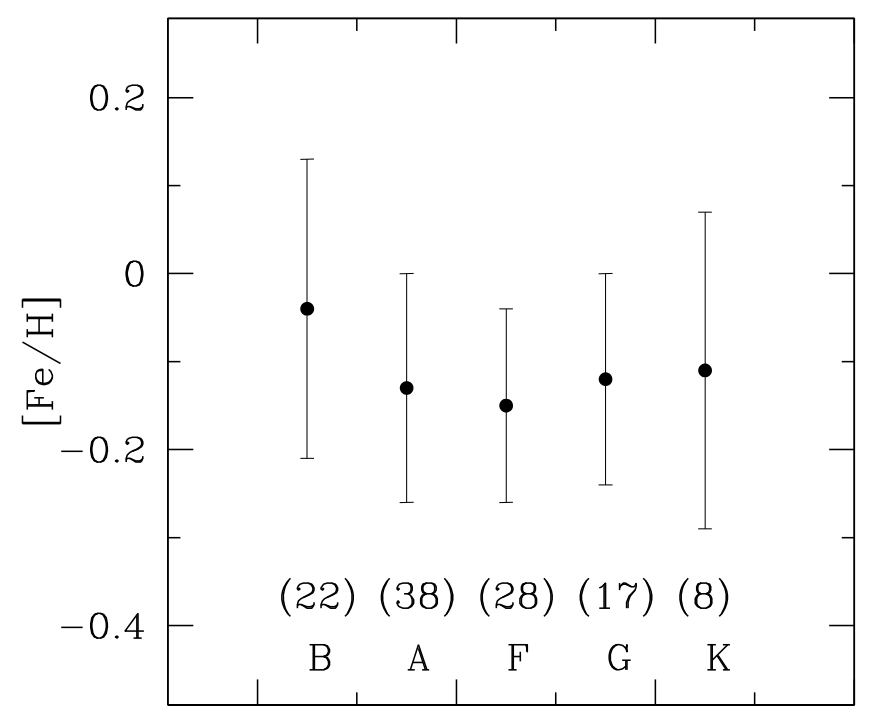

\section{Spectral Types}

Fig. 6. Metallicity of Vega-like stars of different spectral types. Between brackets is indicated the number of objects in each spectral type bin. The vertical lines are the corresponding dispersions.

metal content of the disk favors the fast formation of giant planets, which needs to accrete an atmosphere and migrate inward before the gas is dissipated from the disk. On the contrary, for Vega-like objects no giant planet needs to be formed and/or migrate inward. The gas may dissipate and still the planetesimal in the external part of the disk may produce dust by collisions.

We tentatively analyzed two small sub-sets of Vega-like objects: the Vega-like stars with giant planets and the Vega-like group with no Doppler detected giant planets. The first group is composed of 7 stars: 6 with $70 \mu \mathrm{m}$ excess detected by Spitzer (HD 33636, HD 50554, HD 52265, HD 82943, HD 128311 and 
HD 117176; Beichman et al. 2006a) and $\epsilon$ Eri with infrared and submillimieter excesses (Greaves et al. 1998; Zuckerman 2001). In the second group we include 5 stars without giant exoplanets detected by the Doppler technique (Santos et al. 2004; Gilli et al. 2006) and showing infrared excess in 24 or $70 \mu \mathrm{m}$ (HD 7570, HD 38858, HD 69830, HD 76151 and HD 115617; Beichman et al. 2006a; Bryden et al. 2006). HD 69830 is included in the second group since it harbors 3 Neptune- or super-Earth-mass planets (Lovis et al. 2006).

The median metallicity of Vega-like stars with planets is +0.07 dex and the dispersion is 0.16 dex. For the Vega-like objects without planets these values are: -0.08 and 0.18 dex, respectively. It seems that when a Vega-like star has a giant planet the metallicity increases slightly. However the small number of objects available as well as the dispersions prevent us from giving any statistical significance to this initial trend.

Greaves et al. (2007) proposed that the solid-mass (i.e., metal) content in primordial disks, called $M_{\mathrm{S}}$, is the fundamental parameter that regulates the planet/disk formation. If $M_{\mathrm{S}}$ is small, the star will form a Vega-like disk, while if $M_{\mathrm{S}}$ is larger, a giant planet may be formed. Table 1 of Greaves et al. (2007) shows the range of metallicity and the final configurations (planet+debris, debris, etc.) derived by these authors. The medians of the metallicities of Vega-like stars with and without planets agree with Greaves et al. (2007)'s Table 1. However this can only be considered as an initial trend that needs to be confirmed by increasing the number of Vega-like objects with planets as well as objects known not to harbor Doppler detected planetary mass objects.

Finally, the stellar mass may also play an important role in the occurrence of giant planets. This type of planet seem to be more frequent in intermediate-mass than in low-mass stars (Johnson et al. 2007). Using radial velocity, Lovis \& Mayor (2007) discovered a giant planet orbiting an open cluster star (NGC 2423, No. 3) with $2.4 M_{\odot}$. Johnson et al. (2008) reported the detection of two giant planets orbiting two intermediate-mass mass stars (1.80 and $1.64 M_{\odot}$, respectively), discovered as part as their survey of evolved stars.

\section{Summary and conclusions}

We derived spectroscopic metallicities for a group of 113 Southern Hemisphere Vega-like stars. We applied two methods to determine metallicities: the "classical" WIDTH method and a comparison with the grid of synthetic spectra of Munari et al. (2005) by means of the Downhill algorithm. The later method offers the advantage that the complete profile of the line is used in the metallicity derivation and not only the equivalent width. In addition we estimated smaller uncertainties in the metallicities derived by the Downhill method ( $0.1 \mathrm{dex})$ than with the WIDTH code $(0.2$ dex $)$.

Vega-like stars have metallicities similar to Solar Neighborhood stars without planets or disks and significantly different to the giant exoplanet host stars. This result confirms previous estimations by Greaves et al. (2006) and Chavero et al. (2006), based on comparatively smaller samples.

The low metallicities for the Vega-like group (median = -11 dex) in relation to the exoplanet host stars (median = +0.17 dex, Santos et al. 2004) may indicate that the probability of these stars hosting a giant planet/s of the type detected by radial velocity surveys is also low. However the dispersion of metallicities within the Vega-like stars is also significant $(0.26 \mathrm{dex})$ and thus a fraction of these objects may have metallicities high enough to form giant planets. We caution that exoplanet host stars are mainly of FGK spectral types whereas the bulge of IRAS detected Vega-like stars has A spectral type which are, in general, excluded from radial velocity searches since high precisions are not feasible. In this we are assuming that the probability of an A star being associated with a giant planet depends on the metallicity, as is the case for FGK stars.

We find no trend in the metallicities of Vega-like objects with the spectral type. The Greaves et al. (2006) suggestion makes compatible the relatively high metallicity of giant exoplanet host stars and the solar neighborhood value for Vega-like stars with the core accumulation model of Pollack et al. (1996).

Analyzing two relatively small sub-samples, we find that Vega-like stars with a Doppler detected planet have slightly higher metallicities than Vega-like stars known not to harbor such a planet. However this must be considered only as an initial trend that needs to be confirmed by increasing both samples to achieve a statistically significant result.

Acknowledgements. The authors thank Drs. F. Castelli, P. Bonifacio and L. Sbordone for making their codes available to them.

\section{References}

Aumann, H. H., Beichman, C. A., Gillett, F. C., et al. 1984, ApJ, 278, 23 Apai, D., Janson, M., Moro-Martin, A., et al. 2008, ApJ, 672, 1196 Backman, D. E., \& Paresce, F. 1993, in Protostars and planets III, ed. E. H. Levy, J.I. Lunine, \& M. S. Mathews (Tucson: Univ. Arizona Press), 1253 Beichman, C. A., Bryden, G., Rieke, G. H., et al. 2005, ApJ, 622, 1160 Beichman, C. A., Bryden, G., Stapelfeldt, K. R., et al. 2006a, ApJ, 652, 1674 Beichman, C. A., Tanner, A., Bryden, G., et al. 2006b, ApJ, 639, 1166 Bryden, G., Beichman, C. A., Trilling, D. E., et al. 2006, ApJ, 636, 1098 Castelli, F. 1998, Mem. Soc. Astron. Ital., 69, 165

Castelli, F., Gratton, R. G., \& Kurucz, R. L. 1997, A\&A, 318, 841 Chavero, C., Gómez, M., Whitney, B. A., \& Saffe, C. 2006, A\&A, 452, 921

Chen, C. H., Patten, B. M., Werner, M. W., et al. 2005, ApJ, 634, 1372 Chen, C. H., Sargent, B. A., Bohac, C., et al. 2006, ApJS, 166, 351 Cheng, K. P., Bruhweiler, F. C., Kondo, Y., \& Grady, C. A. 1992, ApJ, 396, 83 Clampin, M., Krist, J. E., Ardila, D. R., et al. 2003, AJ, 126, 385 Cote, J. 1987, A\&A, 181, 77

Cutispoto, G., Pastori, L., Pasquini, L., et al. 2002, A\&A, 384, 491

Cutispoto, G., Tagliaferri, G., de Medeiros, J. R., et al. 2003, A\&A, 397, 987 Decin, G., Dominik, C., Waters, L. B. F. M., \& Waelkens, C. 2003, ApJ, 598, 636

Fajardo-Acosta, S. B., Stencel, R. E., Backman, D. E., \& Thakur, N. 1999, ApJ, 520,215

Fischer, D. A., \& Valenti, J. A. 2005, ApJS, 622, 1102

Friedemann, C., Guertler, J., \& Loewe, M. 1996, A\&AS, 117, 205

Fuhr, J. R., Martin, G. A., \& Wiese, W. L. 1988, Atomic transition probabilities, Scandium through Manganese, New York: Amer. Instit. Phys. (AIP) and American Chemical Society

Gillett, F. C. 1986, in Light on dark matter; Proceedings of the First InfraRed Astronomical Satellite Conference, Noordwijk, Netherlands, June 1014, 1985 (A87-11851 02-90) (Dordrecht: D. Reidel Publishing Co.), 61 Gillon, M., \& Magain, P. 2006, A\&A, 448, 341

Domingo, A., \& Figueras, F. 1999, A\&A, 343, 446

Gilli, G., Israelian, G., Ecuvillon, A., Santos, N. C., \& Mayor, M. 2006, A\&A, 449,723

Glebocki, R., \& Stawikowski, A. 2000, Acta Astron., 50, 509

González, G. 1998, A\&A, 334, 221

González, G. 1999, MNRAS, 308, 447

González, G., Laws, C., Tyagi, S., \& Reddy, B. E. 2001, AJ, 121, 432

Gray, R. O., Graham, P. W., \& Hoyt, S. R. 2001, AJ, 121, 2159

Greaves, J. S., Holland, W. S., Moriarty-Schieven, G., et al. 1998, ApJ, 506, 133 Greaves, J. S., Fischer, D. A., \& Wyatt, M. C. 2006, MNRAS, 366, 283

Greaves, J. S., Fischer, D. A., Wyatt, M. C., Beichman, C. A., \& Bryden, G. 2007, MNRAS, 378, L1

Habing, H. J., Dominik, C., Jourdain de Muizon, M., et al. 2001, A\&A, 365, 545 Hauck, B., \& Mermilliod, M. 1998, A\&AS, 129, 431

Hekker, S., \& Meléndez, J. 2007, A\&A, 475, 1003

Hillenbrand, L. A., Carpenter, J. M., Kim, J. S., et al. 2008, ApJ, 677, 630

Holland, W. S., Greaves, J. S., Zuckerman, B., et al. 1998, Nature, 392, 788

Jaschek, M., Jaschek, C., \& Egret, D. 1986, A\&A, 158, 325

Johansson, S. 1978, Phys. Scrip., 18, 217

Johnson, J. A., Fischer, D. A., Marcy, G. W., et al. 2007, ApJ, 665, 785 
Johnson, J. A., Marcy, G. W., Fischer, D. A., et al. 2008, ApJ, 675, 784

Jura, M., Chen, C. H., Furlan, E., et al. 2004, ApJS, 154, 453

Kim, J. S., Hines, D. C., Backman, D. E., et al. 2005, A\&A, 632, 659

Kurucz, R. L. 1988, Trans. IAU, XXB, ed. M. McNally (Dordrecht: Kluwer), 168

Kurucz, R. L. 1992, Rev. Mex. Astron. Astrofis., 23, 45

Kurucz, R. L. 1993, ATLAS, Stellar Atmosphere Programs and $2 \mathrm{~km} \mathrm{~s}^{-1}$ grid, Kurucz CM-ROM No. 13, Smithsonian Astrophysical Observatory, Cambridge, MA

Kurucz, R. L. 1994, ATLAS Atomic Data for Fe and Ni, Kurucz CM-ROM No. 22, Smithsonian Astrophysical Observatory, Cambridge, MA

Lastennet, E., Lignies, F., Buser, R., et al. 2001, The Journal of Astronomical Data, 7

Laureijs, R. J., Jourdain de Muizon, M., Leech, K., et al. 2002, A\&A, 387, 285

Laws, C., González, G., Walker, K. M., et al. 2003, AJ, 125, 2664

Lovis, C., \& Mayor, M. 2007, A\&A, 472, 657

Lovis, C., Mayor, M., Pepe, F., et al. 2006, Nature, 441, 305

Mamajek, E. E., Hines, D. C., Backman, D. E., et al. 2005, A\&AS, 207, 6348

Mannings, V., \& Barlow, M. J. 1998, ApJ, 497, 330

Meyer, M. R., Hillenbrand, L. A., Backman, D. E., et al. 2004, ApJS, 154, 422

Moore, C. E. 1945, A multiplet table of astrophysical interest, Princeton, N.J., The Observatory, Rev. ed.

Mora, A., Merín, B., Solano, E., et al. 2001, A\&A, 378, 116

Munari, U., Sordo, R., Castelli, F., \& Zwitter, T. 2005, A\&A, 442, 1127

Napiwotzki, R., Schoenberner, D., \& Wenske, V. 1993, A\&A, 268, 653

Nissen, P. E. 1988, A\&A, 199, 146

Nordström, B., Mayor, M., Andersen, J., et al. 2004, A\&A, 418, 989

Oudmaijer, R. D., van der Veen, W. E. C. J., Waters, L. B. F. M., et al. 1992, A\&AS, 96, 625

Pascucci, I., Gorti, U., Hollenbach, D., et al. 2006, ApJ, 651, 1177

Pasquini, L., Döllinger, M. P., Weiss, A., et al. 2007, A\&A, 473, 979

Patten, B. M., \& Willson, L. A. 1991, AJ, 102, 323

Pizzolato, N., Maggio, A., Micela, G., Sciortino, S., \& Ventura, P. 2003, A\&A, 397,147

Press, W. H., Teukolsky, S. A., Vetterling, W. T., \& Flannery, B. P. 1992, Numerical Recipes in Fortran: The Art of Scientific Computing (Cambridge University Press), 2nd edition, 617

Pollack, J. B., Hubickyj, O., Bodenheimer, P., et al. 1996, Icarus, 124, 62

Qiu, H. M., Zhao, G., Chen, Y. Q., \& Li, Z. W. 2001, ApJ, 548, 953

Reader, J., Corliss, C. H., Wiese, W. L., \& Martin, G. A. 1980, Wavelengths and transition probabilities for atoms and atomic ions - Part 1: Wavelengths,
National Standard Reference Data Series (NSRDS-NBS), Washington: National Bureau of Standards (NBS)

Reiners, A. 2006, A\&A, 446, 267

Rieke, G. H., Su, K. Y. L., Stansberry, J. A., et al. 2005, ApJ, 620, 1010 Rogers, N. Y. 1995, Comm. Asteroseism., 78

Royer, F., Gerbaldi, M., Faraggiana, R., \& Gómez, A. E. 2002, A\&A, 381, 105

Ruland, F., Biehl, D., Holweger, H., \& Griffin, R. 1980, A\&A, 92, 70

Sadakane, K., \& Nishida, M. 1986, PASP, 98, 685

Sadakane, K., Ohkubo, M., Takeda, Y., et al. 2002, PASJ, 54, 911

Saffe, C., \& Levato, H. 2004, A\&A, 418, 1083

Santos, N. C., Israelian, G., \& Mayor, M. 2000, A\&A, 363, 228

Santos, N. C., Israelian, G., \& Mayor, M. 2001, A\&A, 373, 1019

Santos, N. C., Israelian, G., Mayor, M., Rebolo, R., \& Udry, S. 2003, A\&A, 398, 363

Santos, N. C., Israelian, G., \& Mayor, M. 2004, A\&A, 415, 1153

Santos, N. C., Israelian, G., Mayor, M., et al. 2005, A\&A, 437, 1127

Schneider, G., Silverstone, M. D., Hines, D. C., et al. 2006, ApJ, 650, 414

Sheret, I., Ramsay Howat, S. K., \& Dent, W. R. F. 2003, MNRAS, 343, 65

Sheret, I., Dent, W. R. F., \& Wyatt, M. C. 2004, MNRAS, 348, 128

Silverstone, M. D., Meyer, M. R., Mamajek, E. E., et al. 2006, ApJ, 639, 1138

Stencel, R. E., \& Backman, D. E. 1991, ApJS, 75, 905

Song, I., Caillault, J.-P., Barrado y Navascués, D., \& Stauffer, J. R. 2001, ApJ, 546,352

Sousa, S. G., Santos, N. C., Mayor, M., et al. 2008, A\&A, in press

Strom, S. E., Wolff, S. C., \& Dror, D. H. A. 2005, ApJ, 129, 809

Su, K. Y. L., Rieke, G. H., Stansberry, J. A., et al. 2006, ApJ, 653, 675

Sylvester, R. J., \& Mannings, V. 2000, MNRAS, 313, 73

Sylvester, R. J., Skinner, C. J., Barlow, M. J., \& Mannings, V. 1996, MNRAS, 279,915

Takeda, Y. 1992, A\&A, 253, 487

Telesco, C. M., Fisher, R. S., Piña, R. K., et al. 2000, ApJ, 530, 329

Thevenin, F., \& Idiart, T. P. 1999, ApJ, 521, 753

Trilling, D. E., Bryden, G., Beichman, C. A., et al. 2008, ApJ, 674, 1086

Udry, S., Mayor, M., Benz, W., et al. 2006, A\&A, 447, 361

Uzpen, B., Kobulnicky, H. A., Olsen, K. A. G., et al. 2005, ApJ, 629, 512

Walker, H. J., \& Wolstencroft, R. D. 1988, PASP, 100, 1509

Waters, L. B. F. M., van den Ancker, M. E., Baas, F., et al. 1995, A\&A, 299, 173

Wyatt, M. C., Smith, R., Su, K. Y. L., et al. 2007a, ApJ, 663, 365

Wyatt, M. C., Clarke, C. J., \& Greaves, J. S. 2007b, MNRAS, 380, 1737

Yudin, R. V. 2001, A\&A, 368, 912

Zuckerman, B. 2001, A\&ARv, 39, 549 
Table 1. Sample of Vega-like stars observed at the CASLEO.

\begin{tabular}{|c|c|c|c|c|c|}
\hline Star & $\begin{array}{c}\text { Distance } \\
{[\mathrm{pc}]}\end{array}$ & 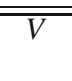 & $\begin{array}{r}v \sin i \\
{\left[\mathrm{~km} \mathrm{~s}^{-1}\right]}\end{array}$ & $\begin{array}{c}\text { Spectral } \\
\text { type }\end{array}$ & Reference \\
\hline HD 105 & 40 & 7.51 & 14.5 & G0V & DEC03 HILL08 \\
\hline HD 142 & 26 & 5.70 & 11.0 & G1V & BE05 TR08 \\
\hline HD 2623 & 365 & 7.93 & 9.0 & $\mathrm{~K} 2$ & SB91 \\
\hline HD 3003 & 46 & 5.07 & 10.4 & $\mathrm{~A} 0 \mathrm{~V}$ & MB98 O92 SB91 WY07 \\
\hline HD 9672 & 61 & 5.62 & 19.3 & A1V & MB98 O92 SB91 PW91 WW88 SN86 WY07 \\
\hline HD 10647 & 17 & 5.52 & 5.5 & F8V & MB98 O92 SB91 DEC03 TR08 \\
\hline HD 10700 & 4 & 3.49 & 5.9 & G8V & MB98 HDJL01 DEC03 \\
\hline HD 10800 & 27 & 5.88 & 4.4 & $\mathrm{G} 2 \mathrm{~V}$ & MB98 BE06 BR06 \\
\hline HD 17206 & 14 & 4.47 & 26.0 & F5V & O92 SB91 \\
\hline HD 17848 & 51 & 5.25 & 22.0 & $\mathrm{~A} 2 \mathrm{~V}$ & MB98 \\
\hline HD 18978 & 26 & 4.08 & 15.6 & $\mathrm{~A} 4 \mathrm{~V}$ & SCBS01 \\
\hline HD 20010 & 14 & 3.80 & 15.0 & F8V & O92 WW88 \\
\hline HD 20794 & 6 & 4.26 & 1.5 & G8V & DEC03 BE06 \\
\hline HD 21563 & 182 & 6.14 & 37.0 & $\mathrm{~A} 4 \mathrm{~V}$ & MB98 \\
\hline HD 22049 & 3 & 3.72 & 6.8 & $\mathrm{~K} 2 \mathrm{~V}$ & SB91 WW88 HDJL01 DEC03 \\
\hline HD 22484 & 14 & 4.29 & 4.0 & F9V & DEC03 TR08 \\
\hline HD 23362 & 309 & 7.91 & 6.0 & K2 & SB91 \\
\hline HD 25457 & 19 & 5.38 & 18.0 & F5V & DEC03 PAS06 HILL08 \\
\hline HD 28375 & 118 & 5.53 & 12.5 & B3V & O92 SB91 TR08 \\
\hline HD 28978 & 125 & 5.67 & 20.7 & $\mathrm{~A} 2 \mathrm{~V}$ & BP93 \\
\hline HD 30495 & 13 & 5.49 & 3.6 & G3V & HDJL01 DEC03 TR08 \\
\hline HD 31295 & 37 & 4.64 & 11.7 & $\mathrm{~A} 0 \mathrm{~V}$ & SN86 WY07 \\
\hline HD 33262 & 12 & 4.71 & 26.6 & F7V & BR06 TR08 \\
\hline HD 33636 & 29 & 7.00 & 5.0 & G0 & BE05 TR08 \\
\hline HD 33949 & 172 & 4.36 & 12.2 & B7V & MB98 O92 SB91 PW91 SN86 \\
\hline HD 35850 & 27 & 6.30 & 5.0 & F7V & DEC03 PAS06 AP08 \\
\hline HD 36267 & 88 & 4.20 & 16.3 & B5V & BP93 \\
\hline HD 37484 & 60 & 7.26 & 5.0 & F3V & PAS06 HILL08 \\
\hline HD 38206 & 69 & 5.73 & 3.4 & $\mathrm{~A} 0 \mathrm{~V}$ & MB98 DEC03 WY07 \\
\hline HD 38385 & 53 & 6.25 & 9.0 & F3V & MB98 \\
\hline HD 38393 & 9 & 3.59 & 10.2 & F7V & MB98 HDJL01 SH03 BE06 \\
\hline HD 38678 & 22 & 3.55 & 23.3 & $\mathrm{~A} 2 \mathrm{~V}$ & MB98 O92 PW91 AP91 C87 HDJL01 DEC03 SU06 WY07 \\
\hline HD 39014 & 44 & 4.34 & 18.9 & A7V & O92 SB91 C87 JU04 \\
\hline HD 39060 & 19 & 3.85 & 13.3 & $\mathrm{~A} 3 \mathrm{~V}$ & MB98 O92 C92 SB91 PW91 AP91 WW88 C87 JJE86 HDJL01 DEC03 WY07 \\
\hline HD 40136 & 15 & 3.71 & 18.4 & F1V & MB98 BE06b \\
\hline HD 41700 & 27 & 6.35 & 15.7 & G0V & DEC03 HILL08 \\
\hline HD 41742 & 27 & 5.93 & 29.0 & F4V & MB98 \\
\hline HD 43955 & 305 & 5.51 & 5.5 & B3V & MB98 \\
\hline HD 66591 & 166 & 4.81 & 7.3 & B3V & MB98 \\
\hline HD 68456 & 21 & 4.74 & 11.9 & F5V & BR06 TR08 \\
\hline HD 69830 & 13 & 5.95 & 1.6 & K0V & MB98 BE06 BR06 \\
\hline HD 71043 & 73 & 5.89 & 2.5 & $\mathrm{~A} 0 \mathrm{~V}$ & WY07 \\
\hline HD 71155 & 38 & 3.91 & 14.3 & $\mathrm{~A} 0 \mathrm{~V}$ & PW91 C87 WY07 \\
\hline HD 75416 & 97 & 5.46 & 4.8 & B9V & MB98 SU06 WY07 \\
\hline HD 76151 & 17 & 6.01 & 4.0 & G3V & BE06 BR06 \\
\hline HD 79108 & 115 & 6.14 & 10.2 & $\mathrm{~A} 0 \mathrm{~V}$ & WY07 \\
\hline HD 80950 & 81 & 5.86 & 15.3 & A0V & MB98 WY07 \\
\hline HD 82943 & 27 & 6.54 & 3.0 & G0 & BE05 TR08 \\
\hline HD 86087 & 98 & 5.71 & 12.0 & $\mathrm{~A} 0 \mathrm{~V}$ & ВР93 \\
\hline HD 88955 & 32 & 3.85 & 10.5 & $\mathrm{~A} 2 \mathrm{~V}$ & MB98 \\
\hline HD 98800 & 47 & 8.89 & 4.6 & K4V & MB98 SB91 WW88 MA05 \\
\hline HD 99211 & 26 & 4.06 & 7.3 & A9V & MB98 \\
\hline HD 102647 & 11 & 2.14 & 12.4 & A3 V & O92 C92 SB91 PW91 WW88 C87 HDJL01 DEC03 \\
\hline HD 105211 & 20 & 4.14 & 7.3 & F2 & BE06b \\
\hline HD 105686 & 101 & 6.16 & 3.2 & $\mathrm{~A} 0 \mathrm{~V}$ & MB98 \\
\hline HD 108257 & 123 & 4.82 & 12.4 & B3Vn & BP93 \\
\hline HD 108483 & 136 & 3.91 & 10.9 & B3V & MB98 \\
\hline HD 109085 & 18 & 4.30 & 7.3 & $\mathrm{~F} 2 \mathrm{~V}$ & MB98 SB91 SH03 BE06b \\
\hline HD 109573 & 67 & 5.78 & 15.2 & $\mathrm{~A} 0 \mathrm{~V}$ & TE00 \\
\hline
\end{tabular}


Table 1. continued.

\begin{tabular}{|c|c|c|c|c|c|}
\hline Star & $\begin{array}{c}\text { Distance } \\
{[\mathrm{pc}]}\end{array}$ & $V$ & $\begin{array}{r}v \sin i \\
{\left[\mathrm{~km} \mathrm{~s}^{-1}\right]}\end{array}$ & $\begin{array}{c}\text { Spectral } \\
\text { type }\end{array}$ & " Reference \\
\hline HD 111786 & 60 & 6.14 & 3.6 & A0 & WY07 \\
\hline HD 113766 & 131 & 7.48 & 5.0 & F5V & MB98 O92 CH06 \\
\hline HD 115617 & 9 & 4.74 & 6.4 & G5V & BR06 \\
\hline HD 115892 & 18 & 2.75 & 9.0 & $\mathrm{~A} 2 \mathrm{~V}$ & MB98 SU06 WY07 \\
\hline HD 117176 & 18 & 4.97 & 4.7 & G5V & BE05 BR06 \\
\hline HD 117360 & 35 & 6.52 & 19.3 & F6V & MB98 \\
\hline HD 121847 & 104 & 5.20 & 17.5 & B8V & MB98 PW91 \\
\hline HD 123160 & & 8.66 & 7.8 & K5 & SB91 \\
\hline HD 124771 & 169 & 5.06 & 22.1 & B4V & MB98 \\
\hline HD 128311 & 17 & 7.48 & 5.7 & K0 & BE05 BE06 \\
\hline HD 131885 & 121 & 6.91 & 10.0 & $\mathrm{~A} 0 \mathrm{~V}$ & MB98 \\
\hline HD 135344 & 78 & 7.91 & 4.5 & F3V & MB98 O92 WW88 \\
\hline HD 136246 & 143 & 7.18 & 2.0 & A1V & WY07 \\
\hline HD 139365 & 136 & 3.66 & 11.0 & $\mathrm{~B} 2.5 \mathrm{~V}$ & MB98 \\
\hline HD 139664 & 18 & 4.64 & 8.8 & F5V & O92 PW91 WW88 HDJL01 DEC03 CH06 BE06b \\
\hline HD 141569 & 99 & 7.11 & 25.8 & B9 & O92 SB91 WW88 JJE86 SH03 CL03 \\
\hline HD 142096 & 109 & 5.04 & 17.0 & B3V & MB98 O92 SB91 \\
\hline HD 142114 & 133 & 4.59 & 27.3 & $\mathrm{~B} 2.5 \mathrm{~V}$ & MB98 O92 \\
\hline HD 142165 & 127 & 5.38 & 23.8 & B5V & MB98 \\
\hline HD 144432 & 253 & 8.19 & 8.5 & F0V & MB98 O92 WW88 \\
\hline HD 145482 & 143 & 4.58 & 18.5 & $\mathrm{~B} 2 \mathrm{~V}$ & MB98 \\
\hline HD 150638 & 240 & 6.46 & 3.5 & B8V & PW91 \\
\hline HD 152391 & 17 & 6.65 & 3.0 & G8V & DEC03 BR06 TR08 \\
\hline HD 158643 & 131 & 4.78 & 1.7 & $\mathrm{~A} 0 \mathrm{~V}$ & O92 \\
\hline HD 158793 & & 8.83 & 22.5 & & BP93 \\
\hline HD 159082 & 152 & 6.42 & 25.3 & B9.5V & ВP93 \\
\hline HD 160691 & 15 & 5.12 & 3.8 & G5V & MB98 BE05 \\
\hline HD 161868 & 29 & 3.75 & 21.7 & A0V & O92 C87 SN86 \\
\hline HD 164249 & 47 & 7.01 & 21.0 & F5V & PW91 DEC03 \\
\hline HD 164577 & 81 & 4.42 & 21.5 & $\mathrm{~A} 2 \mathrm{~V}$ & WA95 \\
\hline HD 165341 & 5 & 4.03 & 14.7 & K0V & DEC03 \\
\hline HD 166841 & 214 & 6.32 & 29.5 & B9V & MB98 \\
\hline HD 169830 & 36 & 5.90 & 4.5 & F8V & BE05 \\
\hline HD 176638 & 56 & 4.74 & 2.0 & $\mathrm{~A} 0 \mathrm{~V}$ & MB98 \\
\hline HD 177817 & 274 & 6.00 & 13.0 & B7V & DEC03 \\
\hline HD 178253 & 40 & 4.11 & 20.5 & $\mathrm{~A} 0 \mathrm{~V}$ & MB98 PW91 \\
\hline HD 181296 & 48 & 5.03 & 21.9 & $\mathrm{~A} 0 \mathrm{~V}$ & ВР93 МВ98 \\
\hline HD 181327 & 51 & 7.04 & 18.0 & F6V & MB98 SCH06 CH06 \\
\hline HD 181869 & 52 & 3.96 & 8.5 & B8V & MB98 WY07 \\
\hline HD 183324 & 59 & 5.79 & 3.6 & $\mathrm{~A} 0 \mathrm{~V}$ & WY07 \\
\hline HD 185507 & 209 & 5.18 & 6.4 & B3V & BP93 FR96 \\
\hline HD 188228 & 33 & 3.97 & 12.0 & $\mathrm{~A} 0 \mathrm{~V}$ & SU06 \\
\hline HD 191089 & 54 & 7.18 & 4.5 & F5V & MB98 HILL08 CH06 \\
\hline HD 198160 & 73 & 5.67 & 13.4 & A2 & RI05 \\
\hline HD 199260 & 21 & 5.70 & 4.3 & F7V & BE06b \\
\hline HD 203608 & 9 & 4.21 & 5.1 & F6V & MB98 BE06 BR06 \\
\hline HD 206893 & 39 & 6.69 & 29.0 & F5V & DEC03 \\
\hline HD 207129 & 16 & 5.57 & 3.5 & $\mathrm{G} 2 \mathrm{~V}$ & MB98 O92 WW88 O86 HDJL01 DEC03 SH03 TR08 \\
\hline HD 209253 & 30 & 6.63 & 16.0 & F7V & DEC03 PAS06 HILL08 \\
\hline HD 216435 & 33 & 6.03 & 5.7 & G3V & BP93 \\
\hline HD 216437 & 27 & 6.04 & 4.0 & G4V & BE06 BR06 \\
\hline HD 216956 & 8 & 1.17 & 9.5 & A3V & MB98 O92 C92 SB91 WW88 C87 HDJL01 DEC03 HO98 \\
\hline HD 221853 & 71 & 7.35 & 8.9 & F0 & DEC03 \\
\hline HD 224392 & 49 & 5.00 & 20.8 & A1V & MB98 O92 \\
\hline
\end{tabular}

Note - The distances, visual magnitudes and spectral sypes are taken from the Hipparcos catalog.

References (alphabetically sorted): AP08 = Apai et al. (2008), BE05 = Beichman et al. (2005), BE06 = Beichman et al. $(2006 \mathrm{a})$, BE06b = Beichman et al. (2006b), BP93 = Backman \& Paresce (1993), BR06 = Bryden et al. (2006), C87 = Cote (1987), C92 = Cheng et al. (1992), CH06 $=$ Chen et al. (2006), CL03 = Clampin et al. (2003), DEC03 = Decin et al. (2003), FR96 = Friedemann et al. (1996), HDJL01 = Habing et al. (2001), HILL08 = Hillenbrand et al. (2008), HO98 = Holland et al. (1998), JJE86 = Jaschek et al. (1986), JU04 = Jura et al. (2004), MA05 = Mamajek et al. (2005), MB98 = Mannings \& Barlow (1998), O92 = Oudmaijer et al. (1992), PAS06 = Pascucci et al. (2006), PW91 = Patten $\&$ Willson (1991), RI05 = Rieke et al. (2005), SB91 = Stencel \& Backman (1991), SCBS01 = Song et al. (2001), SH03 = Sheret et al. (2003), SCH06 = Schneider et al. (2006), SN86 = Sadakane \& Nishida (1986), SU06 = Su et al. (2006), TE00 = Telesco et al. $(2000)$, TR08 = Trilling et al. (2008), WA95 = Waters et al. (1995), WW88 = Walker \& Wolstencroft (1988), WY07 = Wyatt et al. (2007a). 
C. Saffe et al.: Spectroscopic metallicities of Vega-like stars, Online Material p 3

Table 2. List of Fe lines used for the metallicity determinations applying the WIDTH program.

\begin{tabular}{|c|c|c|}
\hline Wavelength & $\overline{l o g} g f$ & Reference \\
\hline \multicolumn{3}{|l|}{$\mathrm{Fe} \mathrm{I}$} \\
\hline 3927.92 & -2.191 & K94 \\
\hline 4063.59 & 0.070 & FMW \\
\hline 4071.74 & -0.022 & FMW \\
\hline 4122.64 & -7.705 & K94 \\
\hline 4132.06 & -0.650 & FMW \\
\hline 4175.64 & -0.670 & FMW \\
\hline 4184.89 & -0.860 & FMW \\
\hline 4202.03 & -0.708 & FMW \\
\hline 4235.94 & -0.341 & FMW \\
\hline 4247.43 & -0.230 & FMW \\
\hline 4250.12 & -0.405 & FMW \\
\hline 4250.79 & -0.710 & FMW \\
\hline 4260.47 & -0.020 & FMW \\
\hline 4271.76 & -0.164 & FMW \\
\hline 4325.76 & -0.010 & FMW \\
\hline 4383.55 & 0.200 & FMW \\
\hline 4404.75 & -0.142 & FMW \\
\hline 4415.12 & -0.615 & FMW \\
\hline 5044.21 & -2.150 & FMW \\
\hline 5247.05 & -4.946 & FMW \\
\hline 5322.04 & -3.030 & FMW \\
\hline 5373.71 & -0.860 & FMW \\
\hline 5560.21 & -1.190 & FMW \\
\hline 5576.09 & -1.000 & FMW \\
\hline 5651.47 & -2.000 & FMW \\
\hline 5806.73 & -1.050 & FMW \\
\hline 5814.81 & -1.970 & FMW \\
\hline 5852.22 & -1.330 & FMW \\
\hline 5856.09 & -1.640 & FMW \\
\hline 6065.48 & -1.530 & FMW \\
\hline 6065.48 & -3.910 & K94 \\
\hline 6105.13 & -2.050 & FMW \\
\hline 6120.25 & -5.950 & FMW \\
\hline 6151.62 & -3.299 & FMW \\
\hline 6157.73 & -1.260 & FMW \\
\hline 6165.36 & -1.550 & FMW \\
\hline 6213.43 & -2.660 & FMW \\
\hline 6229.23 & -2.970 & FMW \\
\hline 6303.46 & -2.660 & FMW \\
\hline 6380.74 & -1.400 & FMW \\
\hline 6430.85 & -2.006 & FMW \\
\hline Fe II & & \\
\hline 3938.29 & -3.890 & FMW \\
\hline 4178.86 & -2.480 & FMW \\
\hline 4491.40 & -2.700 & FMW \\
\hline 4515.34 & -2.480 & FMW \\
\hline 4520.22 & -2.600 & FMW \\
\hline 4541.52 & -3.050 & FMW \\
\hline 4555.89 & -2.290 & FMW \\
\hline 4576.33 & -3.932 & K88 \\
\hline 4620.52 & -3.280 & FMW \\
\hline 4629.34 & -2.370 & FMW \\
\hline 4666.76 & -3.330 & FMW \\
\hline 5234.61 & -3.785 & K88 \\
\hline 5234.62 & -2.050 & FMW \\
\hline 5425.26 & -3.360 & FMW \\
\hline 6247.56 & -2.329 & K88 \\
\hline 6369.46 & -4.253 & K88 \\
\hline 6416.92 & -2.740 & K88 \\
\hline 6432.68 & -1.059 & K88 \\
\hline 6432.68 & -3.708 & K88 \\
\hline 6442.95 & -2.885 & K88 \\
\hline
\end{tabular}

References (alphabetically sorted): FMW = Fuhr et al. (1988), K88 = Kurucz (1988), K94 = Kurucz (1994). 
Table 3. Temperatures, gravities, microturbulent velocities $(\xi)$, metallicities (average values), dispersions $(\delta)$, slope of the $[\mathrm{Fe} / \mathrm{H}]$ vs. equivalent width plot and number of lines $(N)$ used with the WIDTH9 program, applying the N93 and C97 calibrations for the Vega-like sample.

\begin{tabular}{|c|c|c|c|c|c|c|c|c|c|c|c|c|c|}
\hline \multirow[b]{2}{*}{$\begin{array}{l}\text { HD } \\
\text { number }\end{array}$} & \multirow[b]{2}{*}{$\begin{array}{l}T_{\text {eff }} \\
{[\mathrm{K}]}\end{array}$} & \multicolumn{4}{|c|}{$\overline{\mathrm{N} 93}$} & \multicolumn{8}{|c|}{$\overline{\text { C97 }}$} \\
\hline & & $\begin{array}{l}\log g \\
\text { [dex] }\end{array}$ & $\begin{array}{c}\xi \\
{\left[\mathrm{km} \mathrm{s}^{-1}\right]}\end{array}$ & $\begin{array}{r}{[\mathrm{Fe} / \mathrm{H}]} \\
{[\mathrm{dex}]}\end{array}$ & $\begin{array}{c}\delta[\mathrm{Fe} / \mathrm{H}] \\
{[\mathrm{dex}]}\end{array}$ & $\begin{array}{l}\text { Slope } \\
{\left[\mathrm{pm}^{-1}\right]} \\
\times 10^{-3}\end{array}$ & $\begin{array}{l}T_{\text {eff }} \\
{[\mathrm{K}]}\end{array}$ & $\begin{array}{l}\log g \\
{[\operatorname{dex}]}\end{array}$ & $\begin{array}{c}\xi \\
{\left[\mathrm{km} \mathrm{s}^{-1}\right]}\end{array}$ & $\begin{array}{r}{[\mathrm{Fe} / \mathrm{H}]} \\
{[\mathrm{dex}]}\end{array}$ & $\begin{array}{c}\delta[\mathrm{Fe} / \mathrm{H}] \\
{[\mathrm{dex}]}\end{array}$ & $\begin{array}{l}\text { Slope } \\
{\left[\mathrm{pm}^{-1}\right]} \\
\times 10^{-3}\end{array}$ & $N$ \\
\hline 105 & 5914 & 4.57 & 1.43 & -0.37 & 0.26 & 5.0 & 5936 & 4.54 & 1.24 & -0.33 & 0.26 & 2.2 & 15 \\
\hline 142 & 6065 & 4.23 & 1.13 & -0.45 & 0.27 & 3.1 & 6278 & 4.22 & 1.23 & -0.27 & 0.25 & 3.9 & 20 \\
\hline 2623 & 4937 & 4.10 & 0.91 & -0.20 & 0.26 & 2.5 & 4931 & 4.07 & 0.50 & 0.09 & 0.20 & 7.9 & 23 \\
\hline 3003 & 8823 & 4.21 & 1.82 & 0.17 & 0.22 & 2.3 & 8844 & 3.85 & 2.03 & 0.07 & 0.31 & 7.1 & 17 \\
\hline 9672 & 8931 & 4.17 & 1.79 & -0.32 & 0.26 & 5.9 & 8867 & 4.09 & 1.85 & -0.31 & 0.21 & 0.9 & 24 \\
\hline 10647 & 5946 & 4.60 & 1.30 & 0.12 & 0.22 & 7.5 & 5863 & 4.37 & 1.10 & -0.07 & 0.28 & 4.7 & 29 \\
\hline 10700 & 5350 & 5.01 & 0.96 & -0.73 & 0.29 & 4.0 & 5463 & 3.96 & 0.87 & -0.67 & 0.23 & 4.5 & 17 \\
\hline 10800 & 5912 & 4.91 & 1.35 & 0.16 & 0.27 & 6.0 & 5862 & 4.12 & 1.05 & 0.12 & 0.26 & 3.1 & 30 \\
\hline 17206 & 6348 & 4.46 & 1.47 & -0.22 & 0.27 & 8.6 & 6185 & 4.11 & 1.28 & 0.03 & 0.21 & 2.5 & 22 \\
\hline 17848 & 8258 & 4.07 & 2.74 & -0.02 & 0.28 & 5.6 & 8215 & 3.43 & 2.93 & -0.17 & 0.20 & 4.7 & 21 \\
\hline 18978 & 7975 & 4.03 & 3.01 & -0.39 & 0.25 & 2.0 & 8144 & 3.87 & 3.06 & -0.11 & 0.23 & 9.7 & 22 \\
\hline 20010 & 6094 & 4.09 & 1.28 & -0.64 & 0.20 & 6.8 & 6226 & 3.77 & 1.51 & -0.62 & 0.30 & 6.2 & 27 \\
\hline 20794 & 5579 & 4.78 & 0.97 & -0.17 & 0.29 & 3.2 & 5602 & 4.07 & 0.95 & -0.58 & 0.24 & 4.0 & 17 \\
\hline 21563 & 6818 & 4.22 & 1.57 & -0.41 & 0.30 & 4.0 & 6624 & 4.19 & 1.74 & -0.10 & 0.26 & 5.4 & 19 \\
\hline 22049 & 5039 & 3.92 & 0.55 & -0.08 & 0.25 & 4.3 & 4910 & 3.89 & 0.85 & -0.13 & 0.27 & 1.6 & 22 \\
\hline 22484 & 5991 & 4.18 & 1.37 & -0.22 & 0.28 & 6.4 & 5911 & 4.51 & 1.34 & -0.19 & 0.26 & 3.6 & 21 \\
\hline 23362 & 4995 & 4.21 & 0.72 & -0.07 & 0.25 & 4.4 & 4773 & 4.18 & 0.62 & -0.47 & 0.29 & 5.3 & 25 \\
\hline 25457 & 6435 & 4.68 & 1.55 & 0.18 & 0.21 & 5.7 & 6515 & 4.25 & 1.59 & -0.22 & 0.22 & 2.8 & 29 \\
\hline 28375 & 15180 & 4.30 & 0.19 & 0.10 & 0.29 & 6.9 & 15172 & 4.35 & 0.10 & -0.19 & 0.26 & 4.5 & 23 \\
\hline 28978 & 9050 & 4.24 & 1.69 & 0.20 & 0.22 & 8.3 & 9050 & 3.48 & 1.66 & 0.33 & 0.24 & 5.5 & 18 \\
\hline 30495 & 5611 & 4.60 & 1.04 & 0.11 & 0.29 & 2.7 & 5655 & 3.98 & 0.96 & 0.13 & 0.24 & 6.4 & 23 \\
\hline 31295 & 8633 & 4.09 & 2.13 & -0.68 & 0.24 & 2.8 & 8479 & 3.59 & 2.07 & -0.76 & 0.25 & 5.7 & 27 \\
\hline 33262 & 6212 & 4.71 & 1.30 & 0.07 & 0.25 & 4.6 & 6089 & 4.44 & 1.14 & -0.09 & 0.24 & 3.9 & 27 \\
\hline 33636 & 5622 & 4.46 & 1.14 & 0.03 & 0.20 & 7.2 & 5640 & 4.00 & 1.04 & -0.09 & 0.27 & 6.0 & 16 \\
\hline 33949 & 12490 & 3.48 & 0.14 & 0.00 & 0.21 & 3.6 & 12553 & 3.59 & 0.10 & -0.23 & 0.27 & 7.9 & 19 \\
\hline 35850 & 6170 & 4.70 & 1.21 & -0.23 & 0.27 & 2.7 & 6075 & 4.41 & 1.52 & -0.12 & 0.24 & 4.0 & 26 \\
\hline 36267 & 14748 & 4.36 & 0.07 & -0.23 & 0.23 & 2.5 & 14885 & 4.40 & 0.10 & -0.02 & 0.23 & 6.3 & 21 \\
\hline 37484 & 6323 & 4.54 & 1.67 & -0.17 & 0.31 & 2.5 & 6312 & 4.51 & 1.20 & -0.25 & 0.30 & 2.5 & 28 \\
\hline 38206 & 10281 & 4.42 & 0.10 & -0.06 & 0.21 & 2.8 & 10165 & 4.34 & 0.39 & 0.32 & 0.23 & 4.3 & 23 \\
\hline 38385 & 6867 & 3.98 & 1.79 & 0.09 & 0.25 & 2.2 & 6558 & 3.57 & 1.80 & 0.12 & 0.30 & 1.3 & 24 \\
\hline 38393 & 6199 & 4.43 & 1.22 & 0.30 & 0.21 & 4.4 & 6317 & 4.29 & 1.25 & 0.21 & 0.27 & 3.2 & 26 \\
\hline 38678 & 8197 & 4.06 & 2.94 & -0.13 & 0.21 & 6.2 & 8475 & 3.40 & 2.91 & -0.35 & 0.28 & 4.9 & 29 \\
\hline 39014 & 7555 & 3.50 & 2.28 & -0.41 & 0.26 & 6.5 & 7582 & 3.59 & 2.53 & -0.39 & 0.30 & 4.9 & 23 \\
\hline 39060 & 8157 & 4.23 & 3.05 & 0.00 & 0.29 & 5.0 & 7995 & 3.95 & 2.78 & 0.17 & 0.21 & 5.7 & 16 \\
\hline 40136 & 6998 & 4.10 & 1.69 & -0.27 & 0.30 & 7.3 & 6890 & 3.91 & 1.94 & -0.33 & 0.27 & 6.3 & 29 \\
\hline 41700 & 6012 & 4.56 & 1.03 & -0.14 & 0.22 & 4.2 & 5973 & 4.37 & 1.38 & -0.41 & 0.22 & 3.2 & 23 \\
\hline 41742 & 6292 & 4.65 & 1.65 & -0.31 & 0.28 & 9.6 & 6208 & 4.23 & 1.47 & -0.30 & 0.30 & 2.2 & 16 \\
\hline 43955 & 17893 & 4.12 & 0.10 & -0.15 & 0.26 & 4.4 & 17922 & 5.19 & 0.10 & -0.20 & 0.26 & 5.9 & 21 \\
\hline 66591 & 16753 & 4.08 & 0.10 & 0.04 & 0.25 & 6.3 & 16787 & 4.15 & 0.06 & -0.09 & 0.21 & 1.3 & 23 \\
\hline 68456 & 6274 & 4.20 & 1.16 & -0.36 & 0.26 & 9.8 & 6289 & 3.08 & 1.48 & -0.20 & 0.24 & 7.5 & 21 \\
\hline 69830 & 5442 & 5.09 & 0.91 & -0.07 & 0.23 & 7.0 & 5599 & 4.05 & 1.13 & -0.06 & 0.29 & 7.8 & 24 \\
\hline 71043 & 10138 & 4.20 & 0.51 & 0.19 & 0.25 & 8.3 & 10215 & 4.31 & 0.48 & -0.14 & 0.25 & 1.9 & 23 \\
\hline 71155 & 9945 & 4.11 & 0.79 & -0.11 & 0.26 & 2.6 & 9929 & 4.18 & 0.64 & 0.25 & 0.21 & 7.2 & 21 \\
\hline 75416 & 12602 & 4.23 & 0.10 & 0.10 & 0.28 & 8.3 & 12461 & 4.25 & 0.10 & 0.01 & 0.26 & 6.7 & 24 \\
\hline 76151 & 5728 & 4.52 & 0.86 & -0.07 & 0.26 & 9.0 & 5868 & 3.59 & 0.95 & -0.07 & 0.22 & 0.4 & 22 \\
\hline 79108 & 10135 & 4.02 & 0.49 & -0.10 & 0.28 & 3.0 & 10107 & 4.11 & 0.22 & -0.26 & 0.22 & 1.8 & 16 \\
\hline 80950 & 10381 & 4.45 & 0.34 & -0.29 & 0.31 & 5.2 & 10496 & 4.36 & 0.35 & -0.28 & 0.20 & 2.5 & 18 \\
\hline 82943 & 5913 & 4.15 & 1.44 & 0.35 & 0.30 & 5.8 & 5659 & 3.55 & 1.33 & 0.32 & 0.22 & 7.8 & 28 \\
\hline 86087 & 9455 & 4.32 & 1.27 & 0.27 & 0.24 & 1.7 & 9396 & 4.34 & 1.38 & -0.07 & 0.28 & 5.2 & 29 \\
\hline 88955 & 8742 & 4.00 & 2.17 & -0.14 & 0.30 & 4.9 & 8857 & 3.90 & 2.32 & 0.12 & 0.29 & 8.5 & 16 \\
\hline 98800 & 4484 & 3.99 & 0.50 & -0.05 & 0.25 & 5.2 & 4479 & 3.96 & 0.29 & -0.24 & 0.23 & 3.7 & 23 \\
\hline 99211 & 10684 & 3.95 & 0.07 & -0.15 & 0.25 & 5.6 & 10510 & 5.45 & 0.13 & 0.14 & 0.23 & 3.3 & 26 \\
\hline 102647 & 8443 & 4.17 & 2.60 & -0.24 & 0.20 & 1.2 & 8529 & 4.07 & 2.63 & -0.07 & 0.27 & 8.6 & 27 \\
\hline 105211 & 6974 & 4.08 & 1.91 & -0.36 & 0.22 & 2.7 & 6914 & 3.91 & 1.86 & -0.04 & 0.26 & 3.0 & 18 \\
\hline 105686 & 9954 & 4.26 & 0.62 & -0.72 & 0.29 & 2.1 & 9989 & 4.25 & 0.67 & -0.39 & 0.27 & 0.2 & 13 \\
\hline 108257 & 16663 & 4.06 & 0.10 & -0.38 & 0.24 & 6.3 & 16667 & 4.14 & 0.10 & -0.36 & 0.21 & 9.2 & 16 \\
\hline
\end{tabular}


Table 3. continued.

\begin{tabular}{|c|c|c|c|c|c|c|c|c|c|c|c|c|c|}
\hline \multirow[b]{2}{*}{$\begin{array}{l}\text { HD } \\
\text { number }\end{array}$} & \multirow[b]{2}{*}{$\begin{array}{l}T_{\text {eff }} \\
{[\mathrm{K}]}\end{array}$} & \multicolumn{4}{|c|}{ N93 } & \multicolumn{8}{|c|}{ C97 } \\
\hline & & $\begin{array}{l}\log g \\
{[\operatorname{dex}]}\end{array}$ & $\begin{array}{c}\xi \\
{\left[\mathrm{km} \mathrm{s}^{-1}\right]}\end{array}$ & $\begin{array}{r}{[\mathrm{Fe} / \mathrm{H}]} \\
{[\operatorname{dex}]}\end{array}$ & $\begin{array}{c}\delta[\mathrm{Fe} / \mathrm{H}] \\
{[\mathrm{dex}]}\end{array}$ & $\begin{array}{l}\text { Slope } \\
{\left[\mathrm{pm}^{-1}\right]} \\
\times 10^{-3}\end{array}$ & $\begin{array}{l}T_{\text {eff }} \\
{[\mathrm{K}]}\end{array}$ & $\begin{array}{l}\log g \\
{[\operatorname{dex}]}\end{array}$ & $\begin{array}{c}\xi \\
{\left[\mathrm{km} \mathrm{s}^{-1}\right]}\end{array}$ & $\begin{array}{r}{[\mathrm{Fe} / \mathrm{H}]} \\
{[\mathrm{dex}]}\end{array}$ & $\begin{array}{c}\delta[\mathrm{Fe} / \mathrm{H}] \\
{[\mathrm{dex}]}\end{array}$ & $\begin{array}{c}\text { Slope } \\
{\left[\mathrm{pm}^{-1}\right]} \\
\times 10^{-3}\end{array}$ & $N$ \\
\hline 108483 & 20406 & 4.36 & 0.13 & 0.02 & 0.21 & 2.6 & 20338 & 4.55 & 0.19 & 0.14 & 0.25 & 5.4 & 20 \\
\hline 109085 & 6734 & 4.27 & 1.68 & -0.20 & 0.24 & 1.8 & 6611 & 4.08 & 1.41 & -0.23 & 0.27 & 6.4 & 20 \\
\hline 109573 & 9305 & 4.32 & 1.38 & -0.06 & 0.31 & 2.8 & 9437 & 4.36 & 1.54 & 0.10 & 0.30 & 5.7 & 15 \\
\hline 111786 & 8075 & 3.90 & 2.67 & -1.42 & 0.30 & 3.8 & 8090 & 3.84 & 2.71 & -1.65 & 0.22 & 5.4 & 24 \\
\hline 113766 & 6843 & 4.35 & 1.51 & 0.06 & 0.23 & 7.4 & 6870 & 4.17 & 1.57 & -0.14 & 0.27 & 4.6 & 16 \\
\hline 115617 & 5506 & 4.51 & 0.75 & 0.20 & 0.29 & 4.2 & 5427 & 3.54 & 0.81 & 0.13 & 0.24 & 5.3 & 16 \\
\hline 115892 & 8658 & 4.05 & 2.36 & -0.29 & 0.27 & 3.3 & 8526 & 3.66 & 2.43 & -0.33 & 0.28 & 3.2 & 14 \\
\hline 117176 & 5351 & 4.01 & 0.95 & 0.01 & 0.26 & 5.4 & 5348 & 3.28 & 0.77 & -0.12 & 0.26 & 6.5 & 25 \\
\hline 117360 & 6169 & 4.46 & 1.39 & -0.39 & 0.21 & 8.1 & 6275 & 4.28 & 1.10 & -0.61 & 0.26 & 8.3 & 25 \\
\hline 121847 & 12556 & 4.02 & 0.16 & -0.30 & 0.26 & 2.5 & 12461 & 4.07 & 0.10 & 0.10 & 0.27 & 4.4 & 16 \\
\hline 123160 & 4297 & 4.10 & 0.18 & 0.27 & 0.27 & 1.8 & 4465 & 4.07 & 0.50 & 0.17 & 0.25 & 7.3 & 28 \\
\hline 124771 & 16042 & 4.09 & 0.21 & 0.15 & 0.21 & 5.3 & 16133 & 4.16 & 0.10 & 0.01 & 0.21 & 9.2 & 29 \\
\hline 128311 & 4583 & 4.82 & 0.31 & 0.16 & 0.24 & 5.8 & 4763 & 2.94 & 0.66 & 0.04 & 0.29 & 2.3 & 16 \\
\hline 131885 & 9633 & 4.20 & 1.01 & -0.44 & 0.29 & 5.1 & 9817 & 4.17 & 1.23 & -0.19 & 0.28 & 7.1 & 23 \\
\hline 135344 & 6696 & 4.21 & 1.80 & -0.37 & 0.21 & 5.8 & 6657 & 3.94 & 1.49 & -0.41 & 0.28 & 1.4 & 20 \\
\hline 136246 & 9782 & 4.30 & 1.02 & -0.29 & 0.23 & 5.0 & 9903 & 4.27 & 0.88 & -0.49 & 0.28 & 3.5 & 27 \\
\hline 139365 & 18136 & 4.41 & 0.24 & 0.02 & 0.25 & 1.9 & 17920 & 4.30 & 0.23 & 0.35 & 0.29 & 6.5 & 26 \\
\hline 139664 & 6576 & 4.49 & 1.53 & -0.46 & 0.28 & 2.4 & 6578 & 4.28 & 1.73 & -0.08 & 0.26 & 3.1 & 24 \\
\hline 141569 & 9953 & 4.05 & 0.43 & -0.32 & 0.27 & 3.7 & 9826 & 3.96 & 0.43 & -0.01 & 0.20 & 8.7 & 18 \\
\hline 142096 & 17132 & 4.66 & 0.10 & -0.28 & 0.28 & 6.8 & 17201 & 4.62 & 0.25 & -0.16 & 0.23 & 9.2 & 14 \\
\hline 142114 & 18469 & 4.48 & 0.10 & 0.09 & 0.26 & 5.4 & 18262 & 5.33 & 0.10 & 0.06 & 0.26 & 1.1 & 21 \\
\hline 142165 & 14081 & 4.33 & 0.10 & -0.03 & 0.23 & 5.7 & 13955 & 5.77 & 0.10 & 0.10 & 0.30 & 6.7 & 20 \\
\hline 144432 & 6857 & 3.66 & 1.56 & -0.19 & 0.23 & 9.5 & 6924 & 3.63 & 1.81 & -0.13 & 0.28 & 0.4 & 25 \\
\hline 145482 & 19247 & 4.24 & 0.14 & -0.38 & 0.27 & 4.3 & 19331 & 4.68 & 0.10 & -0.19 & 0.25 & 4.2 & 20 \\
\hline 150638 & 12394 & 4.26 & 0.11 & -0.34 & 0.23 & 2.8 & 12520 & 4.28 & 0.10 & -0.52 & 0.22 & 2.8 & 22 \\
\hline 152391 & 5347 & 4.94 & 1.00 & -0.24 & 0.26 & 1.2 & 5263 & 3.55 & 0.91 & -0.26 & 0.31 & 2.2 & 27 \\
\hline 158643 & 9636 & 3.11 & 0.97 & -0.44 & 0.22 & 3.2 & 9667 & 3.32 & 0.86 & -0.20 & 0.30 & 5.6 & 26 \\
\hline 158793 & 9921 & 3.11 & 0.66 & 0.25 & 0.25 & 3.6 & 9862 & 3.32 & 0.87 & 0.37 & 0.24 & 8.3 & 16 \\
\hline 159082 & 10886 & 4.02 & 0.02 & 0.09 & 0.31 & 4.7 & 11064 & 3.97 & 0.19 & -0.22 & 0.24 & 1.7 & 26 \\
\hline 160691 & 5463 & 4.30 & 0.95 & -0.07 & 0.25 & 4.5 & 5662 & 4.27 & 1.15 & -0.06 & 0.21 & 1.1 & 21 \\
\hline 161868 & 8526 & 3.96 & 2.18 & 0.11 & 0.30 & 7.3 & 8677 & 3.79 & 2.40 & -0.26 & 0.28 & 5.3 & 26 \\
\hline 164249 & 6765 & 4.44 & 1.61 & -0.04 & 0.22 & 7.9 & 6738 & 3.09 & 1.76 & -0.04 & 0.23 & 6.2 & 29 \\
\hline 164577 & 9627 & 3.66 & 1.30 & -0.14 & 0.24 & 6.9 & 9790 & 3.53 & 1.08 & -0.37 & 0.24 & 6.7 & 24 \\
\hline 165341 & 5114 & 4.20 & 0.60 & -0.26 & 0.30 & 0.8 & 5082 & 4.17 & 0.84 & -0.38 & 0.26 & 7.2 & 26 \\
\hline 166841 & 10909 & 3.43 & 0.10 & -0.16 & 0.31 & 0.7 & 11033 & 3.33 & 0.14 & 0.05 & 0.27 & 4.7 & 16 \\
\hline 169830 & 6234 & 4.12 & 1.16 & -0.15 & 0.23 & 4.3 & 6261 & 3.80 & 1.55 & 0.31 & 0.25 & 1.6 & 26 \\
\hline 176638 & 10223 & 3.99 & 0.34 & -0.21 & 0.23 & 2.3 & 10155 & 3.97 & 0.13 & -0.05 & 0.28 & 5.7 & 21 \\
\hline 177817 & 12754 & 3.62 & 0.02 & 0.13 & 0.27 & 4.2 & 12537 & 3.77 & 0.10 & 0.04 & 0.21 & 3.0 & 26 \\
\hline 178253 & 8402 & 3.98 & 2.58 & 0.12 & 0.23 & 9.9 & 8273 & 4.35 & 2.37 & -0.26 & 0.22 & 6.3 & 23 \\
\hline 181296 & 9138 & 4.24 & 1.85 & 0.06 & 0.30 & 3.0 & 9045 & 4.31 & 1.77 & 0.14 & 0.28 & 1.0 & 21 \\
\hline 181327 & 6332 & 4.54 & 1.52 & 0.34 & 0.23 & 4.1 & 6561 & 4.19 & 1.51 & 0.24 & 0.28 & 2.5 & 27 \\
\hline 181869 & 12018 & 4.11 & 0.10 & -0.02 & 0.28 & 5.8 & 12049 & 4.11 & 0.16 & -0.03 & 0.29 & 0.7 & 19 \\
\hline 183324 & 10373 & 4.18 & 0.13 & -1.13 & 0.27 & 7.6 & 10224 & 4.17 & 0.35 & -1.29 & 0.30 & 3.5 & 21 \\
\hline 185507 & 21389 & 3.96 & 0.10 & -0.14 & 0.27 & 4.0 & 21481 & 4.59 & 0.10 & -0.07 & 0.21 & 2.0 & 31 \\
\hline 188228 & 10444 & 4.29 & 0.10 & -0.17 & 0.25 & 0.2 & 10312 & 4.23 & 0.28 & -0.02 & 0.31 & 6.4 & 17 \\
\hline 191089 & 6323 & 4.43 & 1.58 & -0.41 & 0.25 & 1.4 & 6332 & 4.09 & 1.55 & -0.17 & 0.29 & 7.8 & 24 \\
\hline 198160 & 7817 & 3.91 & 3.02 & -0.78 & 0.29 & 7.3 & 7928 & 4.02 & 2.92 & -0.99 & 0.24 & 5.4 & 25 \\
\hline 199260 & 6120 & 4.65 & 1.14 & -0.26 & 0.28 & 5.0 & 6134 & 4.37 & 1.38 & -0.01 & 0.31 & 5.1 & 18 \\
\hline 203608 & 6203 & 4.66 & 1.61 & -0.43 & 0.28 & 7.0 & 6107 & 4.42 & 1.58 & -0.72 & 0.24 & 3.7 & 19 \\
\hline 206893 & 6587 & 4.40 & 1.39 & -0.07 & 0.27 & 8.5 & 6366 & 4.37 & 1.35 & 0.20 & 0.23 & 4.3 & 24 \\
\hline 207129 & 5838 & 4.41 & 1.33 & -0.26 & 0.20 & 3.8 & 5899 & 3.65 & 1.35 & -0.22 & 0.27 & 1.0 & 26 \\
\hline 209253 & 6208 & 4.65 & 1.13 & 0.02 & 0.27 & 6.5 & 6044 & 4.40 & 1.58 & -0.26 & 0.26 & 6.8 & 16 \\
\hline 216435 & 5687 & 3.84 & 1.09 & 0.07 & 0.26 & 9.2 & 5899 & 5.23 & 1.27 & -0.17 & 0.25 & 6.5 & 22 \\
\hline 216437 & 5709 & 4.04 & 1.21 & 0.34 & 0.21 & 5.6 & 5727 & 5.37 & 1.18 & 0.27 & 0.28 & 5.9 & 21 \\
\hline 216956 & 8762 & 4.18 & 2.28 & -0.42 & 0.28 & 4.8 & 8729 & 4.87 & 1.84 & -0.22 & 0.20 & 2.0 & 22 \\
\hline 221853 & 6213 & 4.02 & 1.24 & -0.18 & 0.24 & 2.8 & 6082 & 3.99 & 1.48 & 0.08 & 0.26 & 3.5 & 15 \\
\hline 224392 & 8885 & 4.12 & 1.82 & 0.09 & 0.21 & 1.4 & 8712 & 4.10 & 2.09 & 0.14 & 0.27 & 2.7 & 23 \\
\hline
\end{tabular}


C. Saffe et al.: Spectroscopic metallicities of Vega-like stars, Online Material p 6

Table 4. $T_{\text {eff }}, \log g$ and $[\mathrm{Fe} / \mathrm{H}]$ derived using the Downhill method, for the sample of Vega-like stars.

\begin{tabular}{lrrr}
\hline Star & $T_{\text {eff }}[\mathrm{K}]$ & $\log g$ & {$[\mathrm{Fe} / \mathrm{H}]$} \\
\hline HD 105 & 5989 & 4.57 & -0.13 \\
HD 142 & 6182 & 4.12 & -0.21 \\
HD 2623 & 4923 & 4.10 & -0.15 \\
HD 3003 & 8794 & 4.10 & 0.06 \\
HD 9672 & 8865 & 4.21 & -0.12 \\
HD 10647 & 5954 & 4.67 & -0.01 \\
HD 10700 & 5499 & 4.97 & -0.53 \\
HD 10800 & 5901 & 4.84 & 0.09 \\
HD 17206 & 6359 & 4.57 & -0.14 \\
HD 17848 & 8308 & 3.96 & -0.05 \\
HD 18978 & 8050 & 4.14 & -0.25 \\
HD 20010 & 6072 & 4.07 & -0.39 \\
HD 20794 & 5629 & 4.70 & -0.35 \\
HD 21563 & 6714 & 4.22 & -0.25 \\
HD 22049 & 4963 & 3.92 & -0.17 \\
HD 22484 & 5943 & 4.29 & -0.17 \\
HD 23362 & 4899 & 4.21 & -0.23 \\
HD 25457 & 6364 & 4.68 & 0.00 \\
HD 28375 & 15275 & 4.20 & -0.02 \\
HD 28978 & 9075 & 4.26 & 0.17 \\
HD 30495 & 5759 & 4.53 & 0.01 \\
HD 31295 & 8651 & 4.11 & -0.75 \\
HD 33262 & 6073 & 4.83 & -0.10 \\
HD 33636 & 5744 & 4.56 & -0.08 \\
HD 33949 & 12459 & 3.44 & -0.07 \\
HD 35850 & 6021 & 4.66 & -0.05 \\
HD 36267 & 14760 & 4.27 & -0.01 \\
HD 37484 & 6380 & 4.54 & -0.22 \\
HD 38206 & 10135 & 4.36 & 0.14 \\
HD 38385 & 6726 & 3.87 & 0.02 \\
HD 38393 & 6163 & 4.37 & 0.08 \\
HD 38678 & 8327 & 3.97 & -0.19 \\
HD 39014 & 7489 & 3.41 & -0.40 \\
HD 39060 & 8036 & 4.21 & 0.11 \\
HD 40136 & 7007 & 4.12 & -0.32 \\
HD 41700 & 6079 & 4.55 & -0.22 \\
HD 41742 & 6331 & 4.61 & -0.33 \\
HD 43955 & 17890 & 4.12 & -0.15 \\
HD 66591 & 16641 & 4.15 & -0.19 \\
HD 68456 & 6305 & 4.14 & -0.39 \\
HD 69830 & 5586 & 5.15 & 0.16 \\
HD 71043 & 10103 & 4.31 & -0.02 \\
HD 71155 & 9881 & 4.22 & 0.14 \\
HD 75416 & 12603 & 4.25 & 0.16 \\
HD 76151 & 5750 & 4.46 & -0.16 \\
HD 79108 & 10273 & 4.11 & -0.07 \\
HD 80950 & 10330 & 4.36 & -0.05 \\
HD 82943 & 5764 & 4.25 & 0.30 \\
HD 86087 & 9310 & 4.25 & 0.08 \\
HD 88955 & 8707 & 4.04 & -0.02 \\
HD 98800 & 4595 & 3.99 & -0.22 \\
HD 99211 & 10625 & 4.90 & -0.01 \\
HD 102647 & 8522 & 4.26 & -0.25 \\
HD 105211 & 6901 & 3.91 & -0.29 \\
HD 105686 & 9930 & 4.19 & -0.48 \\
HD 108257 & 16576 & 3.98 & -0.53 \\
\hline & 20320 & 4.33 & -0.06 \\
HD 10955 & 9378 & 4.17 & -0.21 \\
HD & 3.84 & -0.03 \\
\hline
\end{tabular}


C. Saffe et al.: Spectroscopic metallicities of Vega-like stars, Online Material $p 7$

Table 4. continued.

\begin{tabular}{lrcr} 
& & & \\
\hline \hline Star & $T_{\text {eff }}[\mathrm{K}]$ & $\log g$ & {$[\mathrm{Fe} / \mathrm{H}]$} \\
\hline HD 113766 & 6796 & 4.32 & 0.09 \\
HD 115617 & 5558 & 4.55 & 0.07 \\
HD 115892 & 8600 & 4.11 & -0.46 \\
HD 117176 & 5495 & 4.02 & -0.08 \\
HD 117360 & 6314 & 4.51 & -0.45 \\
HD 121847 & 12472 & 4.00 & -0.09 \\
HD 123160 & 4356 & 4.10 & 0.04 \\
HD 124771 & 16136 & 4.18 & -0.02 \\
HD 128311 & 4635 & 4.71 & -0.04 \\
HD 131885 & 9680 & 4.20 & -0.23 \\
HD 135344 & 6692 & 4.11 & -0.20 \\
HD 136246 & 9790 & 4.30 & -0.28 \\
HD 139365 & 17990 & 4.33 & 0.17 \\
HD 139664 & 6693 & 4.55 & -0.31 \\
HD 141569 & 9963 & 4.11 & -0.07 \\
HD 142096 & 17034 & 4.75 & -0.27 \\
HD 142114 & 18429 & 4.42 & 0.23 \\
HD 142165 & 14077 & 4.31 & 0.11 \\
HD 144432 & 6957 & 3.55 & -0.18 \\
HD 145482 & 19214 & 4.32 & -0.24 \\
HD 150638 & 12453 & 4.16 & -0.42 \\
HD 152391 & 5418 & 5.05 & -0.12 \\
HD 158643 & 9772 & 3.12 & -0.25 \\
HD 158793 & 9781 & 3.03 & 0.32 \\
HD 159082 & 10990 & 3.91 & -0.06 \\
HD 160691 & 5600 & 4.30 & 0.09 \\
HD 161868 & 8567 & 3.98 & -0.06 \\
HD 164249 & 6620 & 4.32 & -0.09 \\
HD 164577 & 9687 & 3.67 & -0.29 \\
HD 165341 & 5153 & 4.20 & -0.32 \\
HD 166841 & 10885 & 3.36 & -0.02 \\
HD 169830 & 6349 & 4.08 & 0.08 \\
HD 176638 & 10095 & 4.10 & -0.21 \\
HD 177817 & 12667 & 3.72 & 0.19 \\
HD 178253 & 8448 & 4.01 & -0.11 \\
HD 181296 & 9207 & 4.30 & 0.17 \\
HD 181327 & 6449 & 4.44 & 0.29 \\
HD 181869 & 12100 & 4.00 & 0.18 \\
HD 183324 & 10325 & 4.17 & -1.24 \\
HD 185507 & 21374 & 4.59 & 0.04 \\
HD 188228 & 10366 & 4.23 & -0.13 \\
HD 191089 & 6402 & 4.33 & -0.34 \\
HD 198160 & 7860 & 4.02 & -1.03 \\
HD 199260 & 6231 & 4.37 & -0.11 \\
HD 203608 & 6105 & 4.61 & -0.51 \\
HD 206893 & 6454 & 4.40 & -0.05 \\
HD 207129 & 5776 & 4.39 & -0.12 \\
HD 209253 & 6175 & 4.62 & -0.17 \\
HD 216435 & 5755 & 3.82 & -0.17 \\
HD 216437 & 5757 & 3.99 & 0.20 \\
HD 216956 & 8743 & 4.09 & -0.34 \\
HD 221853 & 6196 & 4.02 & 0.00 \\
HD 224392 & 8778 & 4.06 & 0.07 \\
\hline & & & \\
& & &
\end{tabular}

